\title{
On Some Nonlinear Phenomena in the Ionospheric Plasma
}

\author{
P. Caldirola and O. De Barbieri \\ Istituto di Scienze Fisiche dell 'Universita di Milano, Milano, Italy
}

(Received May 25, 1964)

\begin{abstract}
In the first part of the paper we calculate and discuss the distribution function of the electrons of a slightly ionized plasma under the influence of an external magnetic field and of an e.m. wave of the type $\mathbf{E}=\mathbf{E}_{0} \cos \omega t$.

It is shown that, taking into account both elastic and inelastic collisions between electrons and molecules, it is possible to calculate explicitly the mean electronic energy due only to the absorption of the extraordinary wave of the electric field and that it is maximum at the gyromagnetic resonance $\left(\omega=\omega_{H}=\frac{e \mathcal{H}}{m c}\right)$. The study of the effects of inelastic collisions shows that in order to have an equal electronic temperature, the intensity of the electric field must be, in the case of inelastic collisions, about five times greater than that for elastic collisions only.

In the second part of the paper we calculate, for the wave $\mathbf{E}=\mathbf{E}_{0} \cos \omega t$, the components of the complex dielectric permittivity tensor, that are given by expressions dependent in a rather complicated way on $\mathbf{E}_{0}$. It is shown that if $\mathbf{E}_{0}$ is sufficiently small they can be simplified and, using a suitable perturbation technique, it is possible to calculate a nonlinear dispersion relation. This relation contains explicitly $\mathbf{E}_{0}$, and, for $\mathbf{E}_{0} \rightarrow 0$, becomes the AppletonHartree formula. Furthermore, it shows that in the nonlinear case, too, the electric field is split up into two components whose propagation is never independent.

In the third part of the paper we calculate and study the electronic distribution function and the complex dielectric permittivity tensor for a wave of the type $\mathbf{E}=\mathbf{E}_{0}[1+\eta \cos (\alpha t+\beta)]$ $\cos \omega t$ with $\omega \simeq \omega_{H} \gg \alpha$.
\end{abstract}

\section{Introduction}

The study of nonlinear propagation of electromagnetic waves in plasma has recently aroused the interest of many scientists. In general, the methods used in describing the process of propagation of electromagnetic waves in plasma are based on a procedure of linearization of the equations that give the mathematical representation of the physical problem. A very important physical characteristic of the aforesaid nonlinear effects rests on the fact that they can be produced by relatively small electric fields. This can be understood quite easily if we consider a slightly ionized plasma in which the electron-electron and electron-ion collision frequency is much less than the electron-molecule collision frequency. In fact, the propagation of an electromagnetic wave in a plasma causes a relatively high increase of the kinetic energy of the electrons. This is mainly due to two facts: the first is the quite large value of the mean free path $\lambda$ of the electrons in the plasma (so that they can acquire considerable energy from the wave between two collisions), the second is that due to the smallness of the ratio $\delta=\frac{2 m}{M} \simeq 3.4 \cdot 10^{-5}$ between the mass of the electron and the mean mass of the molecules, the mean energy transferred in a collision from the electrons to the neutral component of the plasma is almost negligible. As a final result we see that the energy distribution of the electrons is altered (with an increase of their temperature and mean energy). This variation depends on the quantities $\mathbf{E}, \mathbf{H}, \omega$ that characterize the wave in the plasma. So that the parameters that characterize the plasma from the electromagnetic viewpoint (as the dielectric permittivity $\epsilon_{i k}$, the susceptibility $\chi_{\imath k}$, the conductivity $\sigma_{i k}$ and so on) will depend on these parameters (E and $\omega$ mainly) and on the parameters that characterize the plasma from the kinetic viewpoint (as the mean free path $\lambda$, the collision frequency $\nu$ and so on). In this way in the relations that give the electric polarizability $\mathbf{P}$ or the conduction current density $\mathbf{j}_{c}$ that is

$$
\begin{gathered}
P_{i}=\chi_{i k} E_{k} \\
j_{c_{i}}=\sigma_{i k} E_{k}
\end{gathered}
$$


the electrical parameters $\boldsymbol{\epsilon}_{i k}, \chi_{i k}$, and $\sigma_{i k}$ will depend on $\mathbf{E}$ also and the quantities $P_{i}$ and $j_{c_{i}}$ will no longer be proportional to $\mathbf{E}$. Therefore, the electrodynamic processes in the plasma and the same propagation of electromagnetic waves will become nonlinear. There are two types of approach to the theory of nonlinear effects in plasma: the first one is a generalization of the mean free path method and the second one is the statistical method based on Boltzmann equation. The fundamental equation of the first method is Langevin's equation

$$
\frac{d \mathbf{v}}{d t}+\nu \mathbf{v}=\frac{e}{m}\left[\mathbf{E}+\frac{\mathbf{v}}{c} \times \mathcal{H}\right]
$$

$e, m, \nu, \mathbf{v}$ being respectively charge, mass, collision frequency, and velocity of the electron; $\mathbf{E}$ is the electric field of the radiowave, $\mathcal{H}$ the external magnetic field in which the plasma is immersed. Knowing $\mathbf{E}$, (1) allows us to calculate $\mathbf{v}$, furthermore putting by definition:

$$
\mathbf{j}_{t}=n e \mathbf{v}
$$

where $n$ is the electronic density and $\mathbf{j}_{t}$ is the total current density, it is possible to calculate the conductivity $\sigma_{i k}$ and the dielectric susceptibility $\chi_{i k}$ by means of the equation

$$
\mathbf{j}_{t}=\underline{\underline{\boldsymbol{\sigma}}} \cdot \mathbf{E}+\frac{\partial \mathbf{P}}{\partial t}
$$

In this way are obtained the expressions of the dielectric permittivity $\epsilon_{i k}\left(\epsilon_{i k}=4 \pi\left[\chi_{i k}+\delta_{i k}\right]\right)$ and of the conductivity $\sigma_{i k}$ that must be substituted in the equations of the wave propagation. It is easy to see that these expressions of $\epsilon_{i k}$ and of $\sigma_{i k}$ do not depend on $\mathbf{E}$ and so the propagation, also of a very strong wave, is linear. To overcome this difficulty [Ginzburg and Gurevich, 1960], the electronic collision frequency $\nu$ (that enters into the expressions of $\epsilon_{i k}$ and of $\sigma_{i k}$ ) is considered dependent on the electronic temperature $T_{e}$, e.g., in the following way, ${ }^{1}$

$$
\nu(T)=\nu^{(0)} \sqrt{\frac{T_{e}}{T}}
$$

being $T$ the molecular temperature and $\nu^{(0)}$ the collision frequency in the absence of an electric field $\left(T_{e}=T\right)$. In order to calculate $T_{e}$ a differential equation is established equating $d T_{e} / d t$ to the net power gained by the electron, in the following way:

$$
\frac{d T_{e}}{d t}=\frac{2}{3 k n} \mathbf{j}_{t} \cdot \mathbf{E}-\delta\left(T_{e}-T\right) \nu\left(T_{e}\right)
$$

Given the rather complicated structure of this equation, the usual method to solve it consists in disregarding, at first, the dependence of $\nu$ on $T_{e}$. In the final formulas obtained in this way is introduced $\nu$, given by (2), and so it is possible to calculate $T_{e}$ as a function of the various parameters of the plasma among which is the electric field. In this way $\boldsymbol{\epsilon}_{i k}$ and $\sigma_{i k}$ depend on $\mathbf{E}$ and the equations for the propagation become nonlinear. It is clear that this type of approach to the theory of nonlinear propagation presents defects and contradictions that are typical of the mean free path method. This method, worked out particularly by Townsend and Huxley, is based on the calculation of the distance traveled by the electron under the influence of the accelerating field during the interval of time between two successive collisions. The mean distance traveled by an ensemble of electrons per unit time gives the diffusion velocity of the electrons through the gas. The intensity of the field is always supposed to be weak: the calculation is done by supposing that all the electrons have the same velocity, then the mean value of the results is calculated by means of an electronic distribution function, previously unknown. A maxwellian distribution function is employed to obtain results which do not contain the above mentioned mean values. This method has the drawback of being valid only for weak fields,

1 This dependence is valid for electron-molecule collisions [Ginzburg and Gurevich, 1960]. 
which are not able to deviate the electronic distribution function from its stationary form (maxwellian), without the possibility of exactly defining the general validity and the order of magnitude of the employed approximations. Furthermore, according to Huxley [1937, 1940, 1951, and Huxley and Crompton, 1962,] the method of the mean free paths can be employed whenever we suppose that among all the various electron-molecule types of collision the binary ones are by far the most important and that the motion of the particles can be divided into short periods, in which the collisions occur, separated by comparatively long intervals during which the interactions with the other particles can be neglected in comparison to the action of the field. It is evident that if the behavior of a physical system can be outlined in the above mentioned way, it can be analyzed by means of Boltzmann's integrodifferential equation. From a more general viewpoint, we do not see how it would be possible to base the theory of nonlinear propagation on the equation (1) of dynamies, when we are faced with an electron gas, with a statistical velocity distribution.

\subsection{Fundamental Equations of the Statistical Method}

We are interested in the lower $E$ layer of the ionosphere, i.e., the zone between 80 and 95 $\mathrm{km}$ height. The molecular temperature of this layer varies slowly from $205^{\circ} \mathrm{K}$ (at $80 \mathrm{~km}$ ) to $217^{\circ} \mathrm{K}$ (at $95 \mathrm{~km}$ ). Let us suppose that the temperature gradient is zero and let us take a mean molecular temperature equal to $210^{\circ} \mathrm{K}$. The molecular concentration is of about $10^{14}$ $\mathrm{mol} / \mathrm{cm}^{3}$ in this layer. The electron density varies from $5.10^{2} \mathrm{el} / \mathrm{cm}^{3}$ (at $80 \mathrm{~km}$ ) to $5.10^{4} \mathrm{el} / \mathrm{cm}^{3}$ (at $95 \mathrm{~km}$ ) and therefore it is much inferior to the molecular one. The statistical state of the ionospheric plasma is described by means of two distribution functions: one $f(\mathbf{r}, \mathbf{v}, t)$ for the electrons, the other $F(\mathbf{R}, \mathbf{V}, t)$ for the molecules; these are the solutions of the following system:

$$
\begin{aligned}
& \frac{\partial f}{\partial t}+\mathbf{v} \cdot \nabla_{\mathbf{r}} f+\gamma \cdot \nabla_{\mathbf{v}} f=C_{11}+C_{12} \\
& \frac{\partial F}{\partial t}+\mathbf{V} \cdot \boldsymbol{\nabla}_{\mathbf{R}} F+\boldsymbol{\Gamma} \cdot \boldsymbol{\nabla}_{\mathbf{v}} F=C_{21}+C_{22}^{\gamma}
\end{aligned}
$$

in which $C_{11}$ represents the collision term for electron-electron interaction, $C_{22}$ the collision term for molecule-molecule interactions and last $C_{12}$ and $C_{21}$, the collision terms for electron-molecule interactions and where we have indicated with $\gamma$ and $\boldsymbol{\Gamma}$, the external accelerations acting on the electronic and molecular gases, respectively. Taking into account the characteristics of the ionospheric plasma, the second of the written equations can be simplified in the following way:

$$
C_{22}=0
$$

which, after integration, gives the following (maxwellian) distribution function for the molecular velocities.

$$
F=N\left(\frac{M}{2 \pi k T}\right)^{\frac{3}{2}} \exp \left\{-\frac{M V^{2}}{2 k T}\right\}
$$

where $N$ is the molecular density. Moreover, as $N$ is much greater than $n$ (electronic density), the first equation of the system can be simplified, neglecting $C_{11}$ with respect o $C_{12}$; in this way we obtain:

$$
\frac{\partial f}{\partial t}+\mathbf{v} \cdot \nabla_{\mathbf{r}} f+\frac{e}{m}\left[\mathbf{E}+\frac{\mathbf{v}}{e} \times \mathscr{H}\right] \cdot \nabla_{\mathbf{v}} f=C_{12}
$$

$C_{12}$ is given by the following integral operator that we will indicate with $\boldsymbol{J}\{\boldsymbol{f}\}$

$$
C_{12} \equiv J\{f\}=\iint\left[f\left(\mathbf{v}^{\prime}\right) F\left(\mathbf{V}^{\prime}\right)-f(\mathbf{v}) F(\mathbf{V})\right] g \sigma(\vartheta, g) d \mathbf{V} d \Omega
$$

where $g=|\mathbf{v}-\mathbf{V}|$ is the modulus of the relative velocity, $\sigma(\vartheta, g)$ is the differential cross section, 
$\vartheta$ the scattering angle, $\mathbf{v}^{\prime}$ and $\mathbf{V}^{\prime}$ are the velocities of the electron and of the molecule before the collision (after the collision they become $\mathbf{v}$ and $\mathbf{V}$ respectively). Taking into account the characteristics of the ionospheric plasma, it is possible to simplify (4), [Davydov, 1936 and 1937]. We have already said that $\delta$, the mean energy lost by an electron in a collision, is much less than 1. In the case of elastic collisions,

$$
\delta=\frac{2 m}{M} \simeq 3.4 \cdot 10^{-5}
$$

in the case of inelastic collisions the energy lost by collision is noticeable, but because these collisions are much less frequent than the first ones, the mean value of $\delta$ is always much less than 1. For this reason, the rms velocity of an electron is much greater, also in strong fields, than the mean directed velocity, so that the symmetric portion in $\mathbf{v}$, of the electronic distribution function is much greater than its antisymmetric portion. Moreover, as $\delta$ is small, the collision term (5) can be expressed in differential form; in this way (4) becomes the Boltzmann-FokkerPlanck equation. For these reasons let us develop the electronic distribution function in spherical harmonics in velocity space, putting:

$$
f(\mathbf{r}, \mathbf{v}, t)=f_{0}(\mathbf{r}, v, t)+\mathbf{a}_{v} \cdot \mathbf{f}_{1}(\mathbf{r}, v, t)+\chi(\mathbf{r}, \mathbf{v}, t)
$$

where $\mathbf{a}_{v}=\frac{\mathbf{v}}{\mid \mathbf{v}}$. If we put this equation into (4) and integrate all over $d \Omega$ (differential of the solid angle in velocity space) we obtain:

$$
\frac{\partial f_{0}}{\partial t}+\frac{v}{3} \boldsymbol{\nabla}_{\mathbf{r}} \cdot \mathbf{f}_{1}+\frac{e}{3 m v^{2}} \frac{\partial}{\partial v}\left\{v^{2} \mathbf{E} \cdot \mathbf{f}_{1}\right\}-\frac{\delta}{2 v^{2}} \frac{\partial}{\partial v}\left[\nu v^{3}\left(1+\frac{k T}{m v} \frac{\partial}{\partial v}\right) f_{0}\right]=0 .
$$

Putting again (6), multiplied by $\mathbf{a}_{v}$ into (4) and integrating again all over the solid angle $d \Omega$ we have:

$$
\frac{\partial \mathbf{f}_{1}}{\partial t}+v \nabla_{\mathbf{r}} f_{0}+\frac{e}{m} \frac{\partial f_{0}}{\partial v} \mathbf{E}+\frac{e}{m c} \mathcal{H} \times \mathbf{f}_{1}+\nu \mathbf{f}_{1}=0
$$

where we have put: [Ginzburg and Gurevich, 1960; Davydov, 1937; Chapman and Cowling 1960]:

$$
\begin{aligned}
& J\left\{f_{0}\right\}=\frac{\delta}{2 v^{2}} \frac{\partial}{\partial v}\left[\nu v^{3}\left(1+\frac{k T}{m v} \frac{\partial}{\partial v}\right) f_{0}\right] \\
& J\left\{\mathbf{f}_{1}\right\}=-\nu \mathbf{f}_{1} .
\end{aligned}
$$

In this way, starting from (4), with the approximation (6), we have obtained (7) and (8). To establish these equations we employed the properties of monodromy and orthogonality of $f_{0}$ and $\mathbf{f}_{1}$ expressed by (6) and we supposed that the function $\chi$ and the functions that can be obtained by it with the application of the various operators of (4) are very small in respect to the function $f_{0}$ and of the corresponding ones, obtained by applying to it the various operators of (4). It is easy to see that if the electronic density varies smoothly and if the variation of the electronic current along a mean free path $\lambda$ is small with respect to the product of the electronic density and the velocity, that is if,

then

$$
\lambda\left|\frac{\partial \mathbf{j}}{\partial x}\right|\langle\langle n\langle v\rangle
$$

$$
\chi \simeq \sqrt{\delta}\left|\mathbf{f}_{1}\right| \simeq \delta f_{0}
$$

so that the function $\chi$ is small not only with respect to $f_{0}$ (as we supposed to derive (7) and (8)) but also with respect to $\left|\mathbf{f}_{1}\right|$. It is clear that with the system composed of (7) and (8) it is not possible to determine corrections to $f_{0}$ of the order of $\delta f_{0}$. 


\section{Electronic Distribution Function for a Monochromatic Wave ${ }^{2}$}

Let us suppose that the plasma is homogeneous, in this case (7) and (8) become,

$$
\begin{gathered}
\frac{\partial f_{0}}{\partial t}+\frac{e}{3 m v^{2}} \frac{\partial}{\partial v}\left\{v^{2} \mathbf{E} \cdot \mathbf{f}_{1}\right\}-\frac{\delta}{2 v^{2}} \frac{\partial}{\partial v}\left\{\nu v^{3}\left[1+\frac{k T}{m v} \frac{\partial}{\partial v}\right] f_{0}\right\}=0 \\
\frac{\partial \mathbf{f}_{1}}{\partial t}+\frac{e}{m} \frac{\partial f_{0}}{\partial v} \mathbf{E}+\frac{e}{m c} \mathcal{H} \times \mathbf{f}_{1}+\nu \mathbf{f}_{1}=0 .
\end{gathered}
$$

In order to solve this system of four equations we must define two relaxation times. The first is the relaxation time $\tau_{E}$ of the electric field that is defined as the time necessary to change substantially the field (if $\mathbf{E}=\mathbf{E}_{0} \cos \omega t$ then $\tau_{E} \sim \omega^{-1}$ ). The second is the relaxation time $\tau_{r}$ of the electronic energy; $\tau_{r}$ is of the order of $(\delta \nu)^{-1}$ as can be seen by integrating (3) with $E_{0}=0$. Following Gurevich [1957], we can distinguish two cases: the first in which the electric field varies slowly $\left(\tau_{E}>>\tau_{r}\right)$, the second in which it varies quickly $\left(\tau_{E}<<\tau_{r}\right)$. Now we must consider the case in which the field varies quickly because we study the phenomena related with the propagation of a monochromatic wave:

$$
\mathbf{E}=\mathbf{E}_{0} \cos \omega t
$$

which pulsation is equal, or very near, to the gyropulsation of the medium. In fact the value of the magnetic field of the earth, that enters into (10) varies, in the zone of the ionosphere in which we are interested, between 0.35 Gauss and 0.45 Gauss so that, being $\omega \simeq \omega_{H}, \tau_{E} \simeq 1.4 \cdot 10^{-7}$ sec, because $\omega_{H}=e|\mathcal{H}| / m c ;$ moreover $\tau_{r} \simeq(\delta \nu)^{-1}$ is about $4 \cdot 10^{-3}$ sec, being $\delta \simeq 3.4 \cdot 10^{-5}$ and $\nu \simeq 8 \cdot 10^{6}$ coll/sec. From these calculations we see that in our case $\left(\frac{\tau_{E}}{\tau_{r}}\right)<<1$. Therefore let us develop $f_{0}$ and $\mathbf{f}_{1}$ into a power series of the parameter $\left(\frac{\tau_{E}}{\tau_{r}}\right)$ putting,

$$
\begin{aligned}
& f_{0}=f_{00}+f_{01}+f_{02}+\ldots \\
& \mathbf{f}_{1}=\mathbf{f}_{10}+\mathbf{f}_{11}+\mathbf{f}_{12}+\ldots
\end{aligned}
$$

we find that in the zero order approximation we can neglect the variation of the distribution function due to collisions (of the order of $f / \tau_{r}$ ) in respect to the first term of $(9$ ) (because of the order of $\left.\frac{\partial f}{\partial t} \simeq f / \tau_{E}\right)$ so $\frac{\partial f_{00}}{\partial t}=0$. Therefore,

$$
f_{00}=f_{00}(v)
$$

that is, in the zero order approximation, the symmetric part of the distribution function is independent of time. If we put into (9)

$$
\mathbf{f}_{10}=-\mathbf{u} \frac{\partial f_{00}}{\partial v}
$$

we see that u satisfies Langevin's equation (1). This equation can be solved for $\mathbf{E}$ given by (11) and gives the following expression for the persistent part of $\mathbf{f}_{10}$

$$
\mathbf{f}_{10}=\mathbf{A}(\omega) \frac{\partial f_{00}}{\partial v} \cos \omega t+\mathbf{B}(\omega) \frac{\partial f_{00}}{\partial v} \sin \omega t
$$

2 Some results of this section are already contained in the paper "Sull 'eccitazione dell' "airglow" per mezzo di radioonde", by P. Caldirola, published in Nuovo Cimento Suppl. XIX Series X No. 2 (1961). 
The two vectors $\mathbf{A}(\omega)$ and $\mathbf{B}(\omega)$ are given by:

$$
\left.\begin{array}{rl}
\mathbf{A}(\omega) & =-\frac{e E_{0}}{m} \frac{1}{\left[\left(\omega+\omega_{H}\right)^{2}+\nu^{2}\right]\left[\left(\omega-\omega_{H}\right)^{2}+\nu^{2}\right]}\left\{-\omega_{H}\left(\omega_{H}^{2}-\omega^{2}+\nu^{2}\right) \frac{\mathcal{H} \times \mathbf{E}_{0}}{|\mathcal{H}| E_{0}}\right. \\
& \left.+\nu\left(\omega_{H}^{2}+\omega^{2}+\nu^{2}\right) \frac{\mathbf{E}_{0}}{E_{0}}+\nu\left(\omega_{H}^{2}-3 \omega^{2}+\nu^{2}\right) \frac{\omega_{H}^{2} \cos ^{2} \Phi}{\omega^{2}+\nu^{2}} \frac{\mathcal{H}}{|\mathcal{H}|}\right\} \\
\mathbf{B}(\omega) & =-\frac{e E_{0}}{m} \frac{1}{\left[\left(\omega+\omega_{H}\right)^{2}+\nu^{2}\right]\left[\left(\omega-\omega_{H}\right)^{2}+\nu^{2}\right]}\left\{-2 \omega \omega_{H} \nu \frac{\mathcal{H} \times \mathbf{E}_{0}}{|\mathcal{H}| E_{0}}\right. \\
-\omega\left(\omega_{H}^{2}-\omega^{2}-\nu^{2}\right) & \left.\frac{\mathbf{E}_{0}}{E_{0}}+\omega\left(\omega_{H}^{2}-\omega^{2}+3 \nu^{2}\right) \frac{\omega_{H}^{2} \cos ^{2} \Phi}{\omega^{2}+\nu^{2}} \frac{\mathcal{H}}{|\mathcal{H}|}\right\}
\end{array}\right\}
$$

where $\Phi$ is the angle between $\mathbf{E}_{0}$ and $\mathcal{H}$. Given that $f_{0}(v, t) \simeq f_{00}(v)+f_{01}(v, t)$ and $f_{00}(v)>>f_{01}(v, t)$ we have:

$$
\frac{\partial f_{01}(v, t)}{\partial t}=\frac{\delta}{2 v^{2}} \frac{\partial}{\partial v}\left[\nu v^{3}\left(1+\frac{k T}{m v} \frac{\partial}{\partial v}\right) f_{0}-\frac{2 e}{3 m \delta} v^{2} \mathbf{E} \cdot \mathbf{f}_{10}\right]=\frac{\delta}{2 v^{2}} \frac{\partial \mathscr{f}}{\partial v}
$$

from which

$$
\Delta f_{01}(v, t)=\left\{\frac{\partial}{2 v^{2}} \frac{\partial \mathscr{J}}{\partial v}\right\} t
$$

which is not limited for $t \rightarrow+\infty$. Stipulating then the existence of the electronic distribution function we must impose the condition $\mathscr{F}=0$, that is,

$$
\nu v\left(1+\frac{k T}{m v} \frac{\partial}{\partial v}\right) f_{00}-\frac{2 e}{3 m \delta} \mathbf{E} \cdot \mathbf{f}_{10}=0 .
$$

This is the differential equation for $f_{00}$. Seemingly it is not self-consistent because the time is explicitly contained in the expression $\mathbf{E} \cdot \mathbf{f}_{10}$, but noting that $f_{00}$ must contain only the rms value of the electric field, we obtain,

$$
-\frac{2 e}{3 m \delta}\left\langle\mathbf{E} \cdot \mathbf{f}_{10}\right\rangle \tau_{E}=\nu \frac{e^{2} E_{0}^{2}}{3 m^{2} \delta} \varphi(\nu) \frac{d f_{00}}{d v}
$$

having put

$$
\varphi(\nu)=\frac{\cos ^{2} \Phi}{\omega^{2}+\nu^{2}}+\frac{\sin ^{2} \Phi}{2}\left\{\frac{1}{\left(\omega+\omega_{H}\right)^{2}+\nu^{2}}+\frac{1}{\left(\omega-\omega_{H}\right)^{2}+\nu^{2}}\right\}
$$

so that,

$$
f_{00}=C \exp \left\{-\int_{0}^{v} \frac{m v d v}{k T+\frac{e^{2} E_{0}^{2}}{3 m \delta} \varphi(\nu)}\right\}
$$

where the constant $C$ must be determined from the normalization condition,

$$
\int_{0}^{\infty} 4 \pi v^{2} f_{00}(v) d v=1
$$

We see that the method suggested by Gurevich is particularly clear and precise, because the distribution function in the $n$th approximation is obtained by imposing the condition of boundedness for $t \rightarrow+\infty$ of the subsequent approximation. Keeping in mind that the magnetic field $\mathcal{H}$ produces a plasma anisotropy breaking up the plane polarized electromagnetic wave into two elliptically polarized waves, it is important to get the electronic distribution function for an elliptically polarized electric field $\mathbf{E}$. We express $\mathbf{E}$ in terms of its components along the 
three principal polarization axes,

$$
\mathbf{E}=\mathbf{E}_{\| 0} \cos \omega t+\mathbf{E}_{\perp 0}^{+}+e^{i \omega t}+\mathbf{E}_{\perp 0}^{-} e^{-i \omega t}
$$

where $\mathbf{E}_{\| 0}$ is the plane-polarized field parallel to $\mathcal{H}$ and $E_{\perp 0}^{-}$and $E_{\perp 0}^{+}$are two circularly polarized fields in a plane perpendicular to $\mathcal{H}$ and rotating, respectively, in the same sense or in the opposite sense of the electrons in the magnetic field. For such an electric field the distribution function (15) is still valid provided that $E_{0}^{2} \varphi(\nu)$ is replaced by,

$$
\mathbf{E}_{0}^{2} \varphi(\nu) \rightarrow \frac{E_{\| 0}^{2}}{\omega^{2}+\nu^{2}}+\frac{2 E_{\perp 0}^{+2}}{\left(\omega+\omega_{H}\right)^{2}+\nu^{2}}+\frac{2 E_{\perp 0}^{-2}}{\left(\omega-\omega_{H}\right)^{2}+\nu^{2}}
$$

It is clear that at the gyromagnetic resonance $\left(\omega \sim \omega_{H}\right)$ the energy transferred to the plasma is mainly due to the action of the $E_{\perp 0}^{-}$wave. Therefore, let us consider in $E_{0}^{2} \varphi(\nu)$ only the part due to this wave, in this case (15) becomes,

$$
\begin{aligned}
& f_{00}(v)=C_{00} \exp \left\{-\int_{0}^{v} \frac{m v d v}{k T+\frac{2 e^{2} E_{\perp 0}^{-2}}{3 m \delta} \frac{1}{\left(\omega-\omega_{H}\right)^{2}+v^{2} \lambda^{-2}}}\right\} \\
& =C_{00}\left[1+\frac{v^{2}}{\left[\left(\omega-\omega_{H}\right)^{2}+\frac{2 e^{2} E_{\perp 0}^{-2}}{3 m \delta k T}\right] \lambda^{2}}\right]^{\frac{e^{2} E_{\perp 0}^{-2} \lambda^{2}}{3 \delta k^{2} T^{2}}} \exp \left\{-\frac{m v^{2}}{2 k T}\right\}
\end{aligned}
$$

where we have put,

$$
\nu(v)=\frac{v}{\lambda}
$$

with $\lambda=\left(\pi \mathrm{a}_{0} N\right)^{-1}$ being $\mathrm{a}_{0}$ the "radius" of the molecule, $N$ the molecular density and $\lambda$ the mean free path of the electrons that we take as a constant. According to some authors (Phelps, 1960; Sen and Wyller, 1960] it would be better to choose $\nu$ proportional to $v^{2}$. We have chosen a linear dependence for the greater simplicity of the final formulas obtained, this is tantamount to substituting in them a suitable value $\lambda_{\text {eff }}$, for the mean free path, that takes into account the dependence of $\lambda$ on $v$. Putting,

$$
\gamma=\frac{e^{2} E_{\perp 0}^{-2} \lambda^{2}}{3 \delta k^{2} T^{2}} ; \quad \mu=\frac{m \lambda^{2}}{2 k T}\left(\omega-\omega_{H}\right)^{2},
$$

by means of (17) we can calculate the mean energy $\langle\epsilon\rangle$ absorbed by the electrons of the plasma under the action of the component $E_{\perp 0}^{-}$of the electric field, we get:

$$
\langle\epsilon\rangle=\frac{3}{2} k T(\gamma+\mu)^{1 / 2} \frac{W\left(\frac{\gamma}{2}-\frac{3}{4}, \frac{\gamma}{2}+\frac{5}{4} ; \gamma+\mu\right)}{W\left(\frac{\gamma}{2}-\frac{1}{4}, \frac{\gamma}{2}+\frac{3}{4} ; \gamma+\mu\right)}
$$

where $W(k, m ; z)$ is the Whittaker function [Whittaker and Watson, 1958] of parameters $k$, $m$, and $\operatorname{argument} z$. From (19) we have,

$$
\left(\frac{\partial\langle\epsilon\rangle}{\partial \omega}\right)_{\omega=\omega_{H}}=0
$$

so that the energy absorption is maximal at the perfect resonance, $\omega=\omega_{H}$ (see figs. 1 and 2). 


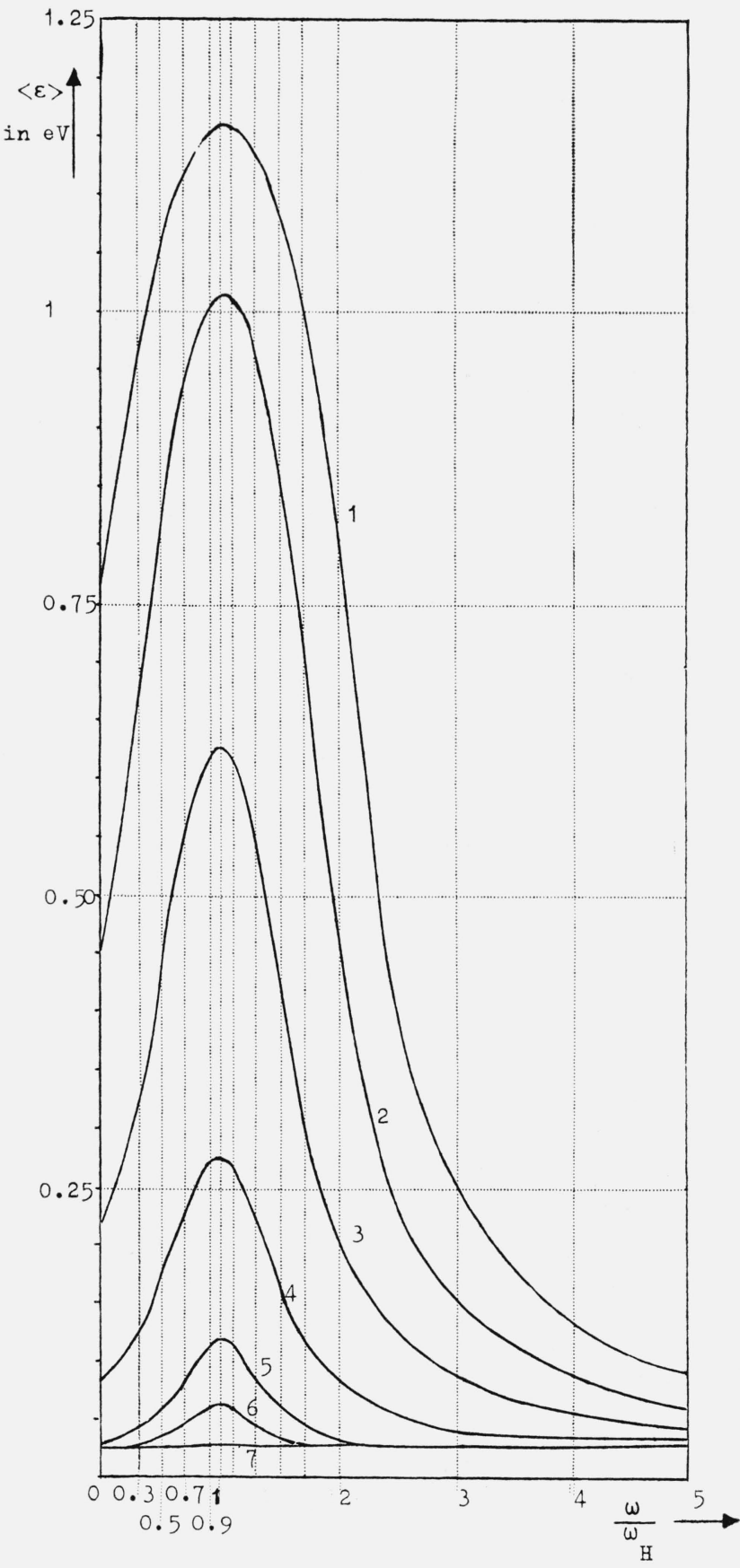

FIGURE 1. Values of $\langle\epsilon\rangle$ calculated by means of (19) in which: $\delta=3.4 \cdot 10^{-5}, \omega_{H}=7.388 \cdot 10^{6}$ puls/sec corresponding to $\mathcal{H}=0.42$ gauss, $\lambda=10 \mathrm{~cm}$ corresponding to a pressure $\mathrm{p}=6.7 \cdot 10^{-3} \mathrm{mmHg}, \mathrm{T}=210{ }^{\circ} \mathrm{K}$.

It is possible to see clearly that the energy absorption maximal for $\omega=\omega_{H}$. Moreover for weak electric fields and for increasing $\frac{\omega}{\omega_{H}}$ all the curves tend to the same horizontal asymptote that is equal to the value of the energy of the electrons in thermal equilibrium with the molecules. The various curves cori espond to the following values of the $E_{\perp 0}$ component of the electric field:

$\begin{array}{lll}\text { Curve 1 } & E_{\perp 0}=10^{-3} & \text { volt } / \mathrm{cm} \\ \text { Curve 2 } & E_{\perp 0}=7.5 \cdot 10^{-4} \text { volt } / \mathrm{cm} \\ \text { Curve 3 } & E_{\perp 0}=5 \cdot 10^{-4} & \text { volt } / \mathrm{cm} \\ \text { Curve 4 } & E_{\perp 0}=2.5 \cdot 10^{-4} \text { volt } / \mathrm{cm} \\ \text { Curve 5 } & E_{\perp 0}=10^{-4} & \text { volt } / \mathrm{cm} \\ \text { Curve 6 } & E_{\perp 0}=5 \cdot 10^{-5} & \text { volt } / \mathrm{cm} \\ \text { Curve 7 } & E_{\perp 0}=10^{-5} & \text { volt } / \mathrm{cm}\end{array}$

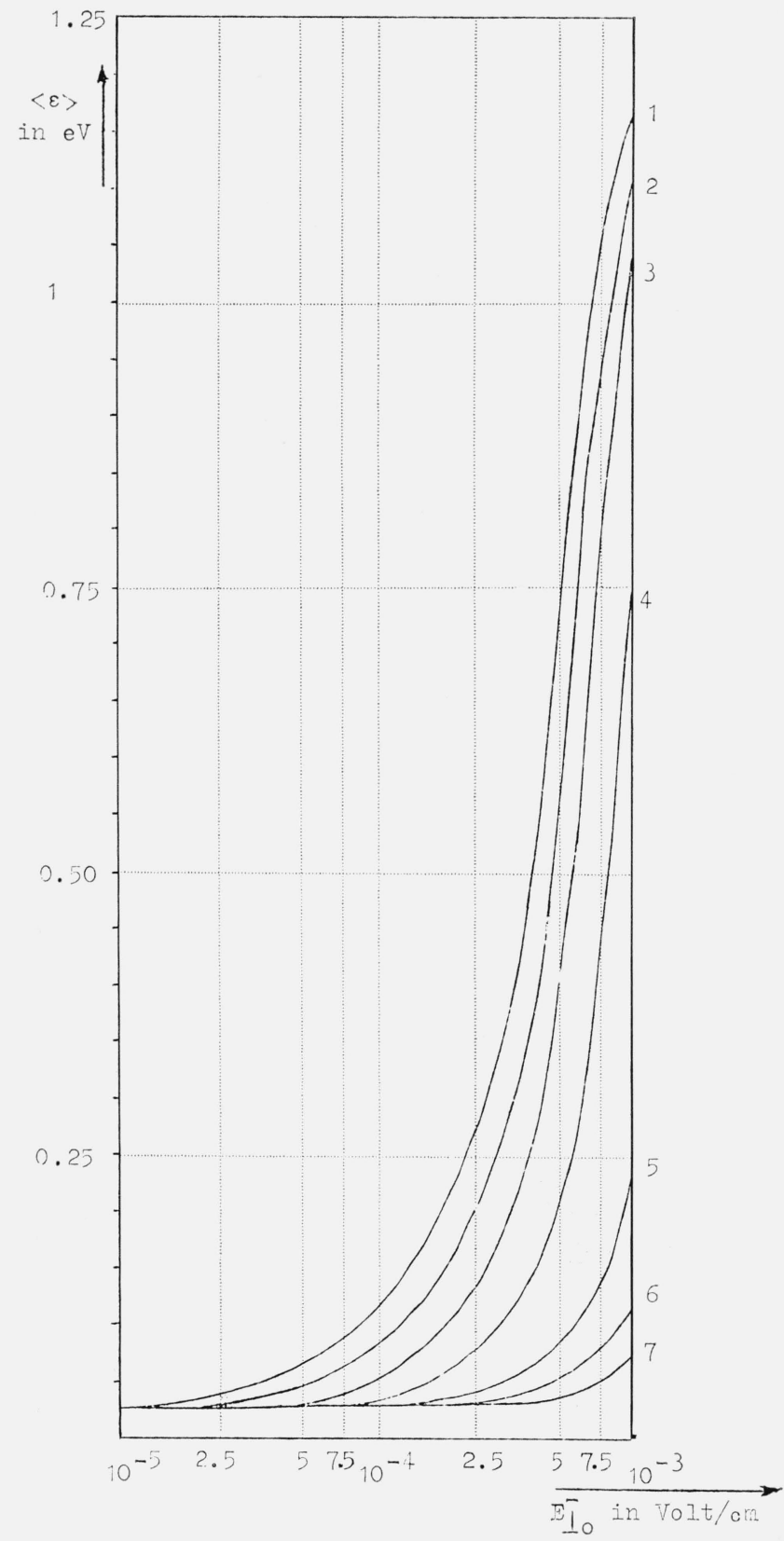

Figure 2. Values of $\langle\epsilon\rangle$ calculated by means of (19) with the same values of the parameters as for figure 1 .

For this figure we can make the same observations as those that we made for figure 1. The various curves correspond to different values of $\frac{\omega}{\omega_{H}}$ namely:

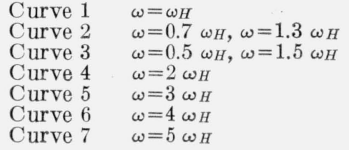

$1.3 \omega_{H}$

Curve $4 \quad \omega=2 \omega_{H}$

Curve $6 \omega=4 \omega$ 
From the asymptotic representation of the Whittaker functions, it is possible to see that

$$
\lim _{\omega \rightarrow \infty}\langle\epsilon(\omega)\rangle=\lim _{\omega_{H} \rightarrow \infty}\left\langle\epsilon\left(\omega_{H}\right)\right\rangle=\frac{3}{2} k T .
$$

From which we see that if the frequency of the wave is far from the gyrofrequency, the electronic distribution function tends to become maxwellian with a temperature equal to the molecular one. At the perfect resonance (17) becomes,

$$
f_{00}^{(r)}(v)=C_{00}^{(r)} \exp \left\{-\int_{0}^{v} \frac{m v d v}{k T+\frac{2 e^{2} E_{\perp 0}^{-2} \lambda^{2}}{3 m \delta v^{2}}}\right\} .
$$

If the electronic temperature is much greater than the molecular one, that is, if

$$
\frac{3}{2} k T<<\frac{e^{2} E_{\perp 0}^{-2} \lambda^{2}}{m \delta v^{2}}
$$

(20) becomes:

$$
f_{00}^{*(r)}(v)=C_{00}^{*(r)} \exp \left\{-\frac{3}{8} \delta\left(\frac{m}{e E_{\perp 0}^{-} \lambda}\right)^{2} v^{4}\right\} .
$$

We have Druyvesteyn's distribution function [Druyvesteyn and Penning, 1940], wherein appears the rms value of the electric field as it should be. With the aid of (21) we can calculate the fraction $P^{*(r)}\left(v \geq v_{0}\right)$ of electrons having a velocity exceeding a certain value $v_{0}$ and the mean energy $\left\langle\epsilon^{*(r)}>\right.$ of the electrons. These quantities are given by,

$$
\begin{gathered}
P^{*(r)}\left(v \geq v_{0}\right)=\frac{W\left(-\frac{1}{8}, \frac{3}{8} ; \frac{3}{8} \delta\left(\frac{m v_{0}^{2}}{e E_{\perp 0}^{-} \lambda}\right)^{2}\right)}{\Gamma\left(\frac{3}{4}\right)\left[\frac{3 \delta}{8}\right]^{3 / 4}\left(\frac{m v_{0}^{2}}{e E_{\perp 0}^{-} \lambda}\right)^{1 / 4} \exp \left\{+\frac{3}{16} \delta\left(\frac{m v_{0}^{2}}{e E_{\perp 0}^{-} \lambda}\right)^{2}\right\}} \\
\left\langle\epsilon^{*(r)}\right\rangle=\frac{\Gamma\left(\frac{5}{4}\right)}{\Gamma\left(\frac{3}{4}\right)} \sqrt{\frac{2}{3 \delta}} e E_{\perp 0}^{-} \lambda .
\end{gathered}
$$

Measuring $\left\langle\epsilon^{*(r)}\right\rangle$ in $\mathrm{eV}, E_{\perp 0}^{-}$in volt $/ \mathrm{cm}, \lambda$ in $\mathrm{cm}$ and the pressure $p$ in $\mathrm{mmHg}$, we have:

$$
\left\langle\epsilon^{*(r)}>=103.58 E_{\perp 0}^{-} \lambda=6.73 \frac{E_{\perp 0}^{-}}{p}\right.
$$

We have found several expressions for the electronic distribution function in a slightly ionized gas under the action of an alternating electric field and a constant magnetic field, considering only elastic collision between electrons and gas molecules. As a result of such calculations [see fig. 3 and eq (23)] it was found that for $\frac{E_{\perp 0}^{-}}{p} \simeq 1$ (that is, for a field intensity of some millivolt per $\mathrm{cm}$ and a pressure of some $10^{-3} \mathrm{mmHg}$ ) it is possible to have electrons accelerated in such a way that their mean energy is several $\mathrm{eV}$ sa that they can collide inelastically with the molecules.

Let us now study the effect of these collisions on the electronic distribution function. In a number of researches (Davydov [1937], Druyvesteyn and Penning [1940], Smit [1936]) approximate methods have been developed in order to take into account this effect and recently a detailed study has been carried out by Kovrizhnykh [1960]. The result achieved by him 
solves, in principle, the problem raised by us, even if the final formulas are generally too cumbersome to be numerically calculated. Due to its laboriousness and to the uncertainty of the experimental data required for its application, Kovrizhnykh's method is difficult to be employed for practical purposes. Consequently, we thought it advisable to adopt, for the evaluation of the effect of inelastic collisions on the electronic velocity distribution, a semi-empirical procedure particularly useful at least as far as our problem is concerned. Let us consider a gas (like air or the ionosphere) consisting essentially of poliatomic molecules. In this gas not only the levels of the electronic configurations but also the rotation and oscillation levels whose energy is rather low (of the order of $\left(10^{-4} \div 10^{-2}\right) \mathrm{eV}$ for rotation levels and $(0.1 \div 0.5) \mathrm{eV}$ for

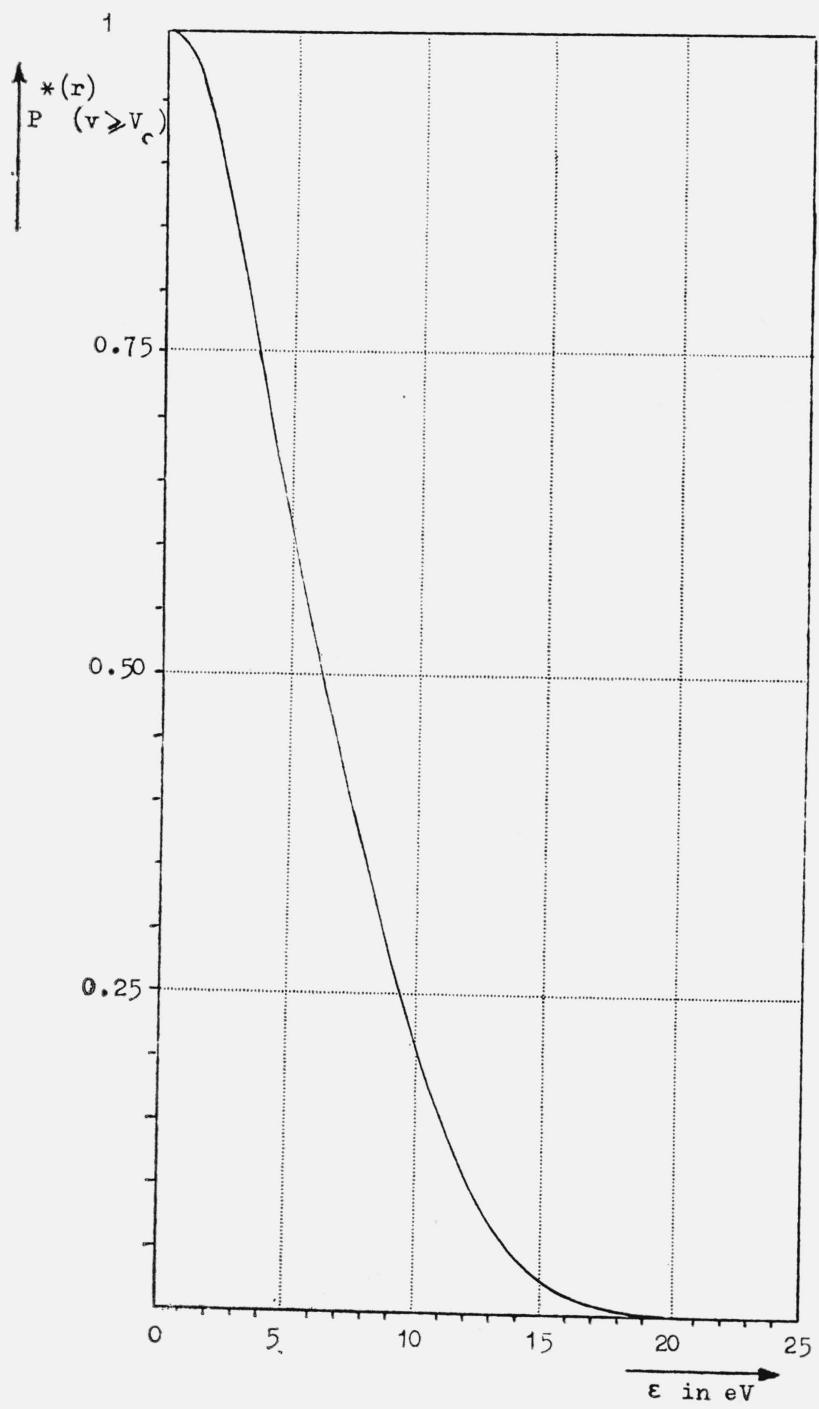

FIGURE 3. Shape of the function $\mathrm{P} *(\mathrm{r}) \quad\left(\mathbf{v}>\mathrm{v}_{\mathbf{0}}\right)$ given by $(22)$ for $\delta=3.4 \cdot 10^{-5}, \mathrm{E}_{\perp_{0}}^{-}=10^{-3}$ volt $/ \mathrm{cm}, \mathrm{p}=10^{-3} \mathrm{mmHg}$ (that is for $\mathrm{E}_{\perp_{0}} / \mathrm{p}=1$ corresponding, according to (23.1), to a mean electronic energy equal to $6.73 \mathrm{eV}), \lambda=66 \mathrm{~cm}$.

From this figure it is possible to see that in this case we are compelled to take into account also of inelastic collisions.

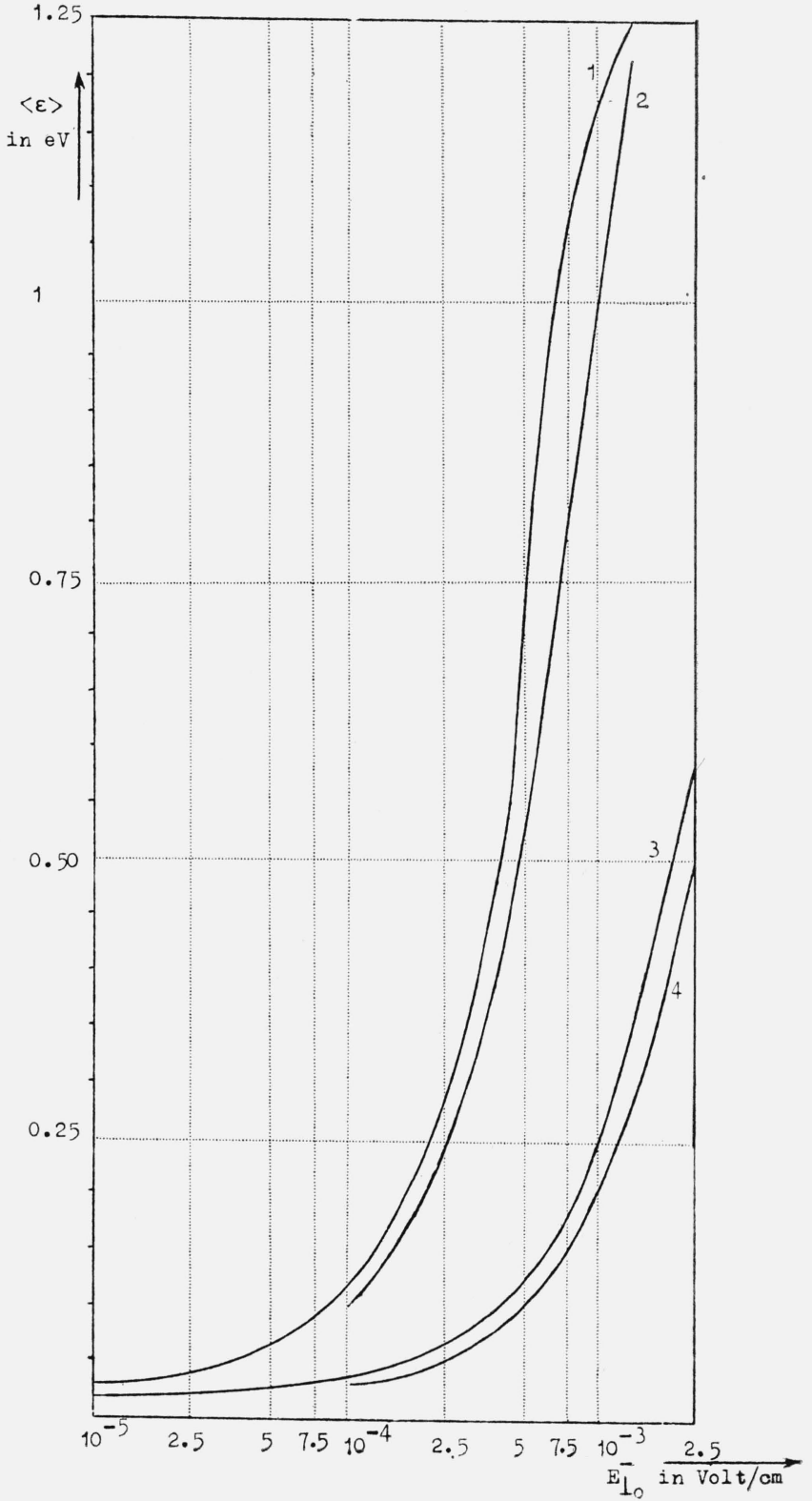

Figure 4. Behavior of $\langle\boldsymbol{\epsilon}\rangle\left(\right.$ for $\left.\omega=\omega_{\mathrm{H}}\right)$ as a function of $\mathrm{E}_{\perp_{\theta}}^{-}$.

Curve 1: values of $\langle\epsilon\rangle$ calculated by means of (19) in which $\delta=3.4 \cdot 10^{-5}$

Curve 2: values of $\left\langle\epsilon>\right.$ calculated by means of (23.1) in which $\delta=3 \cdot 4 \cdot 10^{-5}$

Curve 3: values of $\langle\boldsymbol{\epsilon}\rangle$ calculated by means of $(19)$ in which $\delta=1.4 \cdot 10^{-3}$

Curve 4: values of $\langle\boldsymbol{\epsilon}\rangle$ calculated by means of (23.2) in which $\delta=1.6 \cdot 10^{-3}$ 
oscillation levels) can be excited. Therefore inelastic scattering with molecular excitation takes place also at low temperature and can absorb a considerable amount of electron energy. Keeping in mind the expression of the collision term for elastic collisions,

$$
J^{(\mathrm{el})}\{f\}=+\frac{1}{2 v^{2}} \frac{\partial}{\partial V}\left[v^{2} \delta v\left(\frac{k T}{m} \frac{\partial f}{\partial v}+v f\right)\right]
$$

we write down in the same way the corresponding term for inelastic collisions,

$$
J^{(\text {inel })}\{f\}=+\frac{1}{2 v^{2}} \frac{\partial}{\partial V}\left[v^{2} R(v)\left(\frac{k T}{m} \frac{\partial f}{\partial v}+v f\right)\right]
$$

where $R(v)$ is, corresponding to $\delta v$, the electron energy loss due to inelastic collisions. We can then take into account this term, by substituting in the formulas for the energy distribution of the electrons for elastic collision only, the expression $\delta \nu$ with

$$
R_{\text {tot }}(v)=\delta \nu(v)+R(v)
$$

which corresponds to the introduction of an "efficient" energy loss for collisions given by,

$$
\delta_{(v)}^{(\text {eff })}=\frac{R_{(\text {tot })}(v)}{\nu^{(\text {erf })}(v)} .
$$

The energy loss $\delta^{(e f f)}(v)$ as a function of the electron velocity has been experimentally determined in many papers (for instance [Crompton, Huxley, and Sutton, 1953]). From the experimental data one can deduce that, up to energies of about $2 \mathrm{eV}$, for air and the ionosphere $\delta^{(\text {eff })}$ is nearly constant and in both cases it corresponds to about $1.6 \cdot 10^{-3}$ (to be compared with $\delta=3.4 \cdot 10^{-5}$ for elastic collisions only). Let us now note that in the formulas for electronic energy distribution in the case of elastic collisions only, the parameter $\delta$ appears only through the ratio $E^{2} / \delta$. This leads to the conclusion that, in order to take into account the effect of inelastic collisions, it is sufficient to substitute $E^{2} / \delta$ with $E^{2} / \delta^{(\text {eff })}$ in the final formulas. Thus we can say that the effect of the inelastic collisions is to reduce the value of $E^{2}$ to a value $E^{2}$ (red) such that $E_{(\text {red })}^{2} / \delta=E^{2} / \delta^{(\text {eff })}$. We have then

$$
E_{(\text {red })}=E \sqrt{\frac{\delta}{\delta^{(\text {eff })}}} .
$$

For the air and the ionosphere we shall obtain

$$
E_{(\mathrm{red})}=E \sqrt{\frac{3.4 \cdot 10^{-5}}{1.6 \cdot 10^{-3}}} \simeq \frac{1}{5} E .
$$

The electronic mean energy is therefore reduced (see fig. 4) from its value (23.1) for

$$
\omega=\omega_{H} \frac{3}{2} k T<<\frac{e^{2} E_{\perp 0}^{-2} \lambda^{2}}{m \delta v^{2}}
$$

to

$$
\left\langle\epsilon^{* *(r)}\right\rangle=\frac{1}{5}\left\langle\epsilon^{*(r)}\right\rangle=20.72 E_{\perp 0}^{-} \lambda=1.345 \frac{E_{\perp 0}^{-}}{p} .
$$

\section{Nonlinear Dispersion Relation ${ }^{3}$}

Taking (6) into account we have,

$$
\mathbf{j}_{t}=\left[\underline{\sigma}=i \omega \stackrel{\underline{\underline{\underline{E}}}}{\frac{\underline{\underline{1}}}{4 \pi}}\right] \cdot \mathbf{E}=\frac{4 \pi}{3} e n \int_{0}^{\infty} v^{3} \mathbf{f}_{10} d v .
$$

${ }^{3}$ A. Airoldi of our Institute has contributed to the work contained in this section. We take the occasion to thank her for the aid given in the numerical computations. 
Putting the $z$ axis along the direction of the external magnetic field $\mathcal{H}$ we have,

$$
\left.\begin{array}{l}
\sigma_{z z}=-\frac{\omega_{p}^{2}}{3} \int_{0}^{\infty} v^{3} \frac{\nu}{\omega^{2}+\nu^{2}} \frac{\partial f_{00}}{\partial v} d v \\
\sigma_{x x}=\sigma_{y y}=-\frac{\omega_{p}^{2}}{3} \int_{0}^{\infty} v^{3} \frac{1}{2}\left\{\frac{\nu}{\left(\omega+\omega_{H}\right)^{2}+\nu^{2}}+\frac{\nu}{\left(\omega-\omega_{H}\right)^{2}+\nu^{2}}\right\} \frac{\partial f_{00}}{\partial v} d v \\
\sigma_{x y}=-\sigma_{y x}=-\frac{\omega_{p}^{2}}{3} \int_{0}^{\infty} v^{3} \frac{1}{2}\left\{\frac{\omega+\omega_{H}}{\left(\omega+\omega_{H}\right)^{2}+\nu^{2}}-\frac{\omega-\omega_{H}}{\left(\omega-\omega_{H}\right)^{2}+\nu^{2}}\right\} \frac{\partial f_{00}}{\partial v} d v \\
\sigma_{x z}=\sigma_{z x}=\sigma_{y z}=\sigma_{z y}=0 \\
\frac{\boldsymbol{\epsilon}_{z z-1}}{4 \pi}=\frac{\omega_{p}^{2}}{3} \int_{0}^{\infty} v^{3} \frac{1}{\omega^{2}+\nu^{2}} \frac{\partial f_{00}}{\partial v} d v=\boldsymbol{\epsilon}_{z x}=\boldsymbol{\epsilon}_{y z}=\boldsymbol{\epsilon}_{z y}=0 \\
\frac{\boldsymbol{\epsilon}_{x x-1}}{4 \pi}=\frac{\boldsymbol{\epsilon}_{y y-1}}{4 \pi}=\frac{\omega_{p}^{2}}{3} \int_{0}^{\infty} v^{3} \frac{1}{2 \omega}\left\{\frac{\omega+\omega_{H}}{\left(\omega+\omega_{H}\right)^{2}+\nu^{2}}+\frac{\omega-\omega_{H}}{\left(\omega-\omega_{H}\right)^{2}+\nu^{2}}\right\} \frac{\partial f_{00}}{\partial v} d v \\
\frac{\boldsymbol{\epsilon}_{x y}}{4 \pi}=-\frac{\boldsymbol{\epsilon}_{y x}}{4 \pi}=\frac{\omega_{p}^{2}}{3} \int_{0}^{\infty} v^{3} \frac{1}{2 \omega}\left\{\frac{\nu}{\left(\omega-\omega_{H}\right)^{2}+\nu^{2}}-\frac{\nu}{\left(\omega+\omega_{H}\right)^{2}+\nu^{2}}\right\} \frac{\partial f_{00}}{\partial v} d v
\end{array}\right\}
$$

where $\omega_{p}$ is the plasma frequency, that is,

$$
\omega_{p}^{2}=\frac{4 \pi e^{2} n}{m} .
$$

The knowledge of the expressions of $\epsilon_{i k}$ and $\sigma_{i k}$ allows us to study the problem of the propagation of electromagnetic waves in a plasma. In order to solve this problem, it is possible to use in the linear approximation two methods that, in general, are equivalent. The first consists in the integration of the equations of the wave propagation, the second is related to the calculation and the study of the dispersion relation. The difference between these two methods lies in the fact that: while the first one (the integration of the equations of the wave propagation) allows us to obtain quantitatively exact results only in relatively few cases; the second, on the contrary, gives a much more general description, also if it is only qualitative, of the propagation. More precisely we have that in the case of a uniform plasma the two methods that give equal results, in the nonuniform case the knowledge of the dispersion relation allows us, very often, to write down approximate solutions of the equations of the propagation. These solutions are, in general, the first term of a geometrical optics series. We must stress the fact that if we base the analysis of nonlinear propagation of a monochromatic wave launched in a plasma on (24) we are compelled to admit that the deviations from linearity are not very strong. In fact we must suppose that there is no harmonic generation so that the main effect of nonlinear propagation consists in increasing the absorption coefficient and in modifying the velocity of the wave and not in a substantial variation of the shape of the monochromatic signal (caused by harmonic generation). For these reasons it ought to be possible to write down an approximate solution of the wave equation in the following way,

$$
\mathbf{E}=\mathbf{E}_{0} \exp \left\{i \frac{\omega}{c}\left(c t-\int_{0}^{z} \mathfrak{n} d \zeta\right)\right\}
$$

where now,

$$
\mathfrak{n}=\mathfrak{n}\left(\mathbf{E}_{0}\right)
$$

it is clear that for $E_{0} \rightarrow 0$ this formula must become the Appleton-Hartree formula.

We are now faced with the problem of calculating the integrals (24). If we consider the collision frequency $\nu$ a linear or a quadratic function of the velocity of the electron it is easy to see that, in the case $\mathcal{H} \neq \mathbf{0}$, it is not possible to give closed expressions for the components of 
$\underline{\underline{\epsilon}}$ and $\underline{\underline{\sigma}}$. Furthermore, putting $\nu=$ const., it is possible to calculate all these integrals that are given by expressions that do not contain $\mathbf{E}_{0}$. On the other hand if we evaluate, in this case, the mean energy of an electron we easily arrive at the following formula

$$
\langle\epsilon\rangle=\frac{3}{2} k T\left[1+\frac{e^{2} E_{0}^{2} \varphi(\nu)}{3 m \delta k T}\right]
$$

so that, also if we have a noticeable heating of the plasma, the propagation is linear. All these difficulties can be overcome if we consider only weak fields for which the electronic distribution function does not appreciably deviate from its stationary form (maxwellian). In this way it is possible to calculate this function by developing $f_{00}$ in Taylor series of $E_{0}^{2}$, stopping the expansion after the first two terms and taking then $\nu=$ const. If we indicate the new distribution function obtained in this way with $f\left(v, E_{0}\right)$ we have

$$
f\left(v, E_{0}\right)=f_{00}\left(v, E_{0}=0\right)+\left(\frac{\partial f_{00}}{\partial E_{0}^{2}}\right)_{E_{0}^{2}=0} E_{0}^{2}
$$

that is:

$$
f\left(v, E_{0}\right)=\left(\frac{m}{2 \pi k T}\right)^{\frac{1}{2}}\left[1+\frac{e^{2} E_{0}^{2} \varphi(\nu) v^{2}}{6 \delta(k T)^{2}}\right] \exp \left\{-\frac{m v^{2}}{2 k T}\right\}
$$

the mean energy of an electron calculated by means of this function is given by,

$$
\langle\epsilon\rangle=\frac{3}{2} k T\left[1+\frac{5}{2} a\right]
$$

where,

$$
a=\frac{e^{2} E_{0}^{2} \varphi(\nu)}{2 m \delta k T}
$$

so that we define as a weak field a field satisfying the following inequality, ${ }^{4}$

$$
a \leq \frac{2}{5}
$$

By means of (26.1) it is possible to calculate all the integrals (24) and the following expressions are obtained,

$$
\left.\begin{array}{l}
\sigma_{z z}=\frac{1}{4 \pi} A \omega_{p}^{2} \frac{\nu}{\omega^{2}+\nu^{2}} \\
\sigma_{x x}=\sigma_{y y}=\frac{1}{4 \pi} A \frac{\omega_{p}^{2}}{2}\left[\frac{\nu}{\left(\omega+\omega_{H}\right)^{2}+\nu^{2}}+\frac{\nu}{\left(\omega-\omega_{H}\right)^{2}+\nu^{2}}\right] \\
\sigma_{x y}=-\sigma_{y x}=\frac{1}{4 \pi} A \frac{\omega_{p}^{2}}{2}\left[\frac{\omega+\omega_{H}}{\left(\omega+\omega_{H}\right)^{2}+\nu^{2}}-\frac{\omega-\omega_{H}}{\left(\omega-\omega_{H}\right)^{2}+\nu^{2}}\right] \\
\sigma_{x z}=\sigma_{z x}=\sigma_{y z}=\sigma_{z y}=0 \\
\epsilon_{z z}=1-A \frac{\omega_{p}^{2}}{\omega^{2}+\nu^{2}}=\epsilon_{z x}=\epsilon_{y z}=\epsilon_{z y}=0 \\
\epsilon_{x z}-\epsilon_{y y}=1-A \frac{\omega_{p}^{2}}{2 \omega}\left[\frac{\omega+\omega_{H}}{\left(\omega+\omega_{H}\right)^{2}+\nu^{2}}+\frac{\omega-\omega_{H}}{\left(\omega-\omega_{H}\right)^{2}+\nu^{2}}\right] \\
\epsilon_{x y}=\epsilon_{y x}=A \frac{\omega_{p}^{2}}{2 \omega}\left[\frac{\nu}{\left(\omega+\omega_{H}\right)^{2}+\nu^{2}}-\frac{\nu}{\left(\omega-\omega_{H}\right)^{2}+\nu^{2}}\right]
\end{array}\right\}
$$

1 It must be said that this inequality can be too strong, what is important is that the second term at the right of (27) does not become much greater than the first. 
where:

$$
A=1+a
$$

for $E_{0} \simeq 0$, that is $A=1$, these formulas become those of the linear approximation. Before calculating explicitly the dispersion relation it is better to find the expression of the components of $\underline{\epsilon}$ and $\underline{\sigma}$ in the usual reference system for the study of wave propagation. To this aim let us put the external magnetic field $H$ in the plane $y 0 z$ of the new reference system at an angle $\theta$ with $0 z$ axis. It is easily seen that the matrix that allows us to pass from the old reference system to the new one is given by

$$
\gamma_{i}^{m}=\left|\begin{array}{ccc}
1 & 0 & 0 \\
0 & \cos \theta & \sin \theta \\
0 & -\sin \theta & \cos \theta
\end{array}\right| .
$$

If we indicate with $\epsilon_{i k}^{\prime}$ the components of the complex dielectric permittivity tensor (defined by $\left.\stackrel{\epsilon}{\epsilon}_{i k}^{\prime}=\epsilon_{i k}-i \frac{4 \pi}{\omega} \sigma_{i k}\right)$ in the new reference system and with $\stackrel{\vee}{\epsilon}_{i k}^{\prime}$ the components of the same tensor in the old system, we have

$$
\boldsymbol{\epsilon}_{i k}^{\prime}=\gamma_{i}^{m} \gamma_{k}^{n} \stackrel{\boldsymbol{\epsilon}}{\prime} m n^{\prime}
$$

Introducing for simplicity's sake the notation

we have:

$$
\Omega=\omega-i \nu \quad \alpha=A \frac{\omega_{p}^{2}}{\omega \Omega\left(\Omega^{2}-\omega_{H}^{2}\right)}
$$

$$
\begin{aligned}
& \boldsymbol{\epsilon}_{x x}^{\prime \prime}=1-\alpha \Omega^{2} \\
& \epsilon_{x y}^{\prime}=-\epsilon_{y x}^{\prime}=i \alpha \Omega \omega_{H} \cos \theta \\
& \epsilon_{x z}^{\prime}=-\epsilon_{z x}^{\prime}=i \alpha \Omega \omega_{H} \sin \theta \\
& \epsilon_{y y}^{\prime}=1-\alpha\left(\Omega^{2}-\omega_{H}^{2} \sin ^{2} \theta\right) \\
& \epsilon_{y z}^{\prime}=\epsilon_{z y}^{\prime}=-\alpha \omega_{H}^{2} \sin \theta \cos \theta \\
& \epsilon_{z z}^{\prime}=1-\alpha\left(\Omega^{2}-\omega_{H}^{2} \cos ^{2} \theta\right) .
\end{aligned}
$$

Let us consider the two Maxwell equations:

$$
\begin{aligned}
& \boldsymbol{\nabla} \times \mathbf{H}=\frac{1}{c} \frac{\partial \mathbf{D}}{\partial t}+\frac{4 \pi}{c} \mathbf{j}_{c} \\
& \boldsymbol{\nabla} \times \mathbf{E}=-\frac{1}{c} \frac{\partial \mathbf{H}}{\partial t}
\end{aligned}
$$

for the plane electromagnetic wave (25) propagating along the $z$ axis they give,

$$
\frac{\partial^{2} \mathbf{E}}{\partial z^{2}}-\frac{\omega^{2}}{c^{2}} \stackrel{\epsilon^{\prime}}{=} \cdot \mathbf{E}=0
$$

the two Maxwell equations and (30) are equivalent if we suppose that there is not harmonic generation of the fundamental frequency $\omega$. Furthermore we have,

$$
\begin{aligned}
& \frac{\partial \mathbf{E}}{\partial z}+i \frac{\omega}{c} \mathfrak{n} \mathbf{E}=0 \\
& \frac{\partial^{2} \mathbf{E}}{\partial z^{2}}+\frac{\omega^{2}}{c^{2}} \mathfrak{n}^{2} \mathbf{E}=-i \frac{\omega}{c} \frac{\partial \mathfrak{\imath}}{\partial z} \mathbf{E}
\end{aligned}
$$


but if the electric field is sufficiently weak the propagation will be almost linear so that it ought to be possible to disregard the right member of the last equations; this, on the other hand, agrees with the spirit of the perturbation technique that we have adopted. ${ }^{5}$ So,

$$
\frac{\partial^{2} \mathbf{E}}{\partial z^{2}}+\frac{\omega^{2}}{c^{2}} \mathfrak{n}^{2} \mathbf{E}=0
$$

in this way (30) becomes,

$$
\begin{aligned}
& {\left[\epsilon_{x x}^{\prime}-\mathfrak{n}^{2}\right] E_{0 x}+\epsilon_{x y}^{\prime} E_{0 y}+\epsilon_{x z}^{\prime} E_{0 z}=0} \\
& \epsilon_{y x}^{\prime} E_{0 x}+\left[\epsilon_{y y}^{\prime}-\mathfrak{n}^{2}\right] E_{0 y}+\epsilon_{y z}^{\prime} E_{0 z}=0 \\
& \epsilon_{z x}^{\prime} E_{0 x}+\epsilon_{z y}^{\prime} E_{0 y}+\epsilon_{z z}^{\prime} E_{0 z}=0
\end{aligned}
$$

where, now, this is not a linear homogeneous system in the three unknowns $E_{0 x}, E_{0 y}, E_{0 z}$ as it happens in the linear case, but it is a more complicated system owing to the presence of $E_{0}^{2}$ in the expressions of $\epsilon_{i k}^{\prime}$. However, in this case, too, it is easily shown that there exists a nonzero solution for $E_{0 x}, E_{0 y}, E_{0 z}$ provided that the following determinant is equal to zero:

$$
\left|\begin{array}{lll}
\boldsymbol{\epsilon}_{z x}^{\prime}-\mathfrak{n}^{2} & \epsilon_{x y}^{\prime} & \epsilon_{x z}^{\prime} \\
\epsilon_{y x}^{\prime} & \epsilon_{y y}^{\prime}-n^{2} & \epsilon_{y z}^{\prime} \\
\epsilon_{z x}^{\prime} & \epsilon_{y z}^{\prime} & \epsilon_{z z}^{\prime}
\end{array}\right|=0
$$

from which we have (compare with (6.1) [Budden, 1961]),

being:

$$
\mathrm{n}^{2}=1-\frac{A X}{1-i Z-\frac{Y \sin ^{2} \theta}{2[1-i Z-A X]} \pm \sqrt{\frac{Y^{2} \sin ^{4} \theta}{4[1-i Z-A X]^{2}}+Y^{2} \cos ^{2} \theta}}
$$

being;

$$
X=\frac{\omega_{p}^{2}}{\omega^{2}} \quad Y=\frac{\omega_{H}}{\omega} \quad Z=\frac{\nu}{\omega}
$$

or (compare with (2.3) [Gallet, 1963])

$$
\mathrm{n}^{2}=1-\frac{A b^{2}}{\sigma^{2}-i r \sigma-\sigma \cos \theta\left[B \pm \sqrt{1+B^{2}}\right]}
$$

$$
b=\frac{\omega_{p}}{\omega_{H}}, \quad r=\frac{\nu}{\omega_{H}}, \quad r_{c}=\frac{\sin ^{2} \theta}{2 \cos \theta}, \quad \sigma=\frac{\omega}{\omega_{H}} \quad B=\frac{r_{c} \sigma\left(\sigma^{2}-A b^{2}+i r \sigma\right)}{\left(\sigma^{2}-A b^{2}\right)^{2}+r^{2} \sigma^{2}} .
$$

For $A=1$ (linear case) (31) becomes Appleton-Hartree's formula. In this case, as it is known [Sen and Wyller, 1960], the results obtained by the statistical theory and by the mean free path theory must coincide. The two signs that appear in (31) show that in the nonlinear case too, the field is split up into two components whose propagation is never independent, given that under the square root there appears the total value of the electric field. We will call these two waves ordinary generalized (O.G.) wave and extraordinary generalized (E.G.) wave, identifying them with the corresponding ones of Appleton (O.A. and E.A., respectively) in the limit $E_{0} \rightarrow 0$ for every propagation condition. Let us put

$$
W=\frac{e^{2} E_{0}^{2}}{2 m \delta k T \omega^{2}}
$$

\footnotetext{
${ }^{3}$ It is clear that we can disregard the right hand side of the last equation if $\frac{\omega}{c}\left|\frac{\mathfrak{n}^{1}}{\mathrm{n}^{2}}\right|<<1$; this is the usual condition for the validity of W.K.B. approximation.
} 
and $A$ becomes

$$
A=1+W\left[\frac{\cos ^{2} \Phi}{1+Z^{2}}+\frac{\sin ^{2} \Phi}{2}\left\{\frac{1}{(1+Y)^{2}+Z^{2}}+\frac{1}{(1-Y)^{2}+Z^{2}}\right\}\right]
$$

It is interesting to notice that the electronic density $n$, that is $X$, does not appear in (32) so that all the results that are obtained for the Appleton-Hartree formula when is varied only $n$ are valid also now if we substitute $X$ with $A X$. The zeros of $\mathfrak{n}^{2}$ are located at the points,

$$
A X+i Z=1 \quad A X+i Z=1 \pm Y
$$

and one of the values of $\mathfrak{n}^{2}$ is infinite for,

$$
A X=(1-i Z) \frac{(1-i Z)^{2}-Y^{2}}{(1-i Z)^{2}-Y^{2} \cos ^{2} \theta} .
$$

In this way we see that for $A=1$ we obtain the well known results of the linear theory [Budden, 1961]. Furthermore the two values of $\mathrm{n}^{2}$ are infinite for,

$$
\begin{array}{ll}
Y= \pm(-1 \pm i Z) & \text { if } \sin \Phi \neq 0 \\
Z= \pm i & \text { if } \cos \Phi \neq 0
\end{array}
$$

these poles are also branch points for $n$ if $\theta=\frac{\pi}{2}$. It is easily seen from (15) and (26.1) that in these points we have $f_{00}(v) \equiv 1, f\left(v, E_{0}\right)=\infty$, for these reasons all the integrals (24) diverge. Moreover $A=\infty$ and in this case the perturbation technique that we have used is no more valid. Given that the dispersion relation (31) depends on $\omega$ in a much more complicated way than Appleton-Hartree's formula, we have studied it using an electronic computer. By means of (31) we have calculated the refraction index $\mu$ and the absorption index $\chi$ using the relation,

from which,

$$
\iota^{2}=(\mu-i \chi)^{2}=M-i N
$$

$$
\begin{aligned}
& \mu=\left[\frac{1}{2}\left\{\sqrt{M^{2}+N^{2}}+M\right\}\right]^{1 / 2} \\
& \chi=\left[\frac{1}{2}\left\{\sqrt{M^{2}+N^{2}}-M\right\}\right]^{1 / 2} .
\end{aligned}
$$

In the numerical calculations we have taken as a constant:

(1) The molecular temperature $T, T=200{ }^{\circ} \mathrm{K}$

(2) the external magnetic field $|\mathcal{H}|,|\mathcal{H}|=0.42$ Gauss for which $\omega_{H}=7.388 \cdot 10^{6} \mathrm{puls} / \mathrm{sec}$

(3) the electronic density $n, n=10^{4} \mathrm{el} / \mathrm{cm}^{3}$

(4) the mean energy lost by an electron in a collision, $\delta=1.6 \cdot 10^{-3}$

(5) the direction of the propagation, we have put $\Theta=0$ (longitudinal propagation) in this case

$$
\text { we have, for symmetry, } \Phi=\frac{\pi}{2} \text {. }
$$

We have varied:

(1) The amplitude $E_{0}$ of the electric field

(2) its pulsation around the chosen value of $\omega_{H}$

(3) the electronic collision frequency $\nu$.

The figures show the results that we have obtained.

From the figures 5, 6, and 7 it is possible to see that the absorption index is in the nonlinear case greater than that calculated by means of Appleton-Hartree's formula, moreover, it increases with increasing electric field. For a field equal to, or less than, $10^{-4} \mathrm{volt} / \mathrm{cm}$ we do not have appreciable deviations from the linearity. For low values of the collision frequency (fig. $5, \nu=2.5 \cdot 10^{6} \mathrm{coll} / \mathrm{sec}$ ) the absorption index of the O.G. wave increases very much for $\omega \simeq \omega_{\mathrm{H}}$, with increasing $\nu$ this increase disappears (figs. 6 and 7 ) and for equal $E_{0}$ and $\frac{\omega}{\omega_{H}}$ the ratio between the values of $\chi$ for the O.G. wave (and E.G. wave) and the values of $\chi$ for the O.A. wave (and E.A. wave) tends to 1 with increasing $\nu$. 

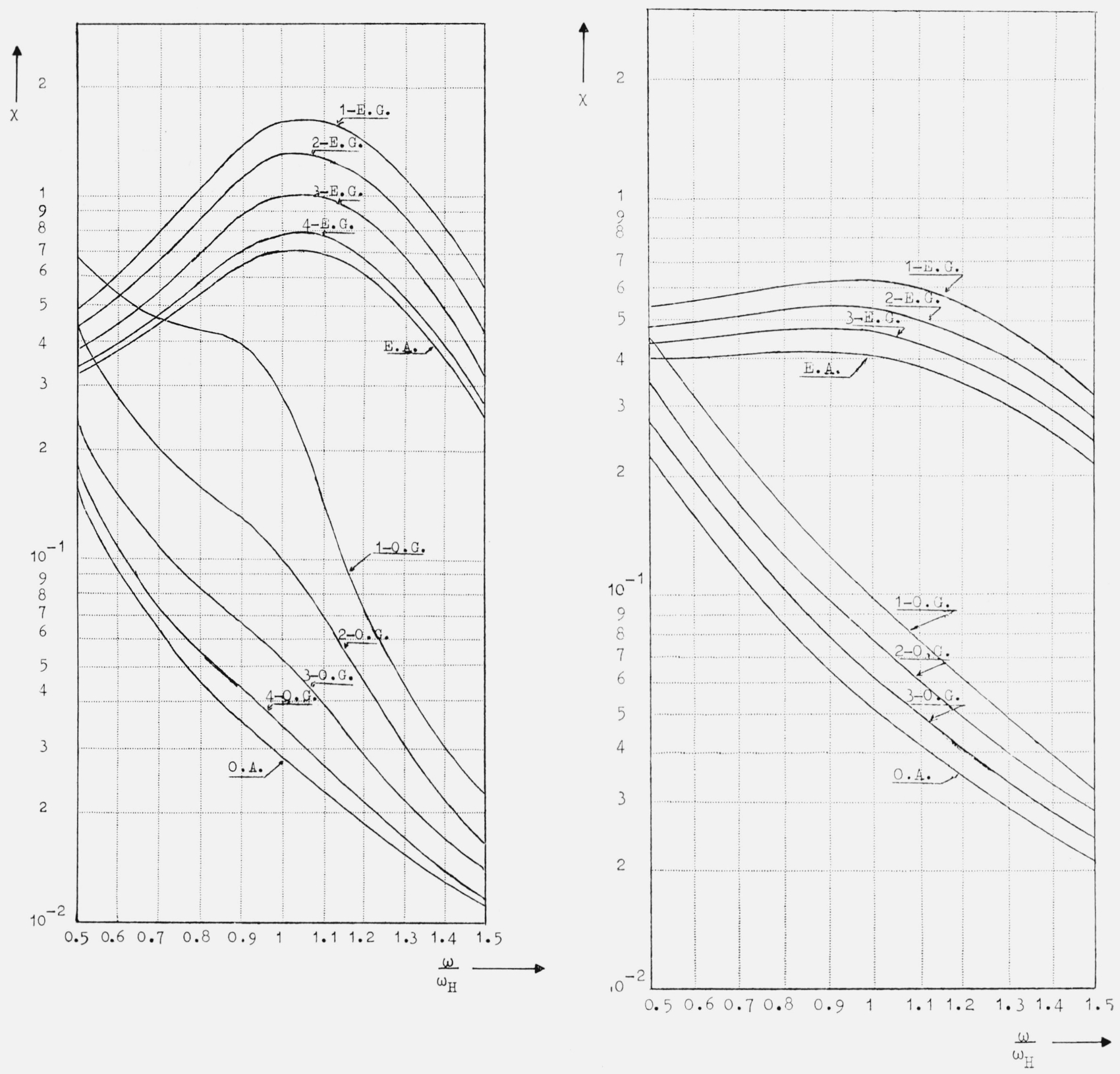

Figure 5. Values of $\chi$ for $\nu=2.5 \cdot 10^{6} \mathrm{coll} / \mathrm{sec}$. Curves 1 (O.G. and E.G.) $E_{0}=10^{-3}$ volt $/ \mathrm{cm} a_{1 \max }=2.49$ Curves 2 (O.G. and E.G.) $E_{0}=7.5 \cdot 10^{-4}$ volt $/ \mathrm{cm} a_{2 \max }=1.40$ Curves 3 (O.G. and E.G.) $E_{0}=5 \cdot 10^{-4}$ volt $/ \mathrm{cm} a_{3 \max }=0.62$

Figure 6. Values of $\chi$ for $\nu=5 \cdot 10^{6} \mathrm{coll} / \mathrm{sec}$.

Curves 4 (O.G. and E.G.) $E_{0}=2.5 \cdot 10^{-4}$ volt $/ \mathrm{cm} a_{4 \max }=0.15$

As it is seen from figures 8,9 , and 10 the behavior of the refraction index $\mu$ is similar to that illustrated for $\chi$ when $E_{0}, \omega, \nu$ are varied.

The fact that the propagation tends to become linear with increasing $\nu$ is easily understandable. In fact if $E_{0}$ and $\omega$ remain fixed the power transferred from the wave to the electrons is unaltered, while with increasing $\nu$ also the power dissipated by the electrons increases due to the collisions, in this way the electronic temperature tends to become equal to the molecular one. 


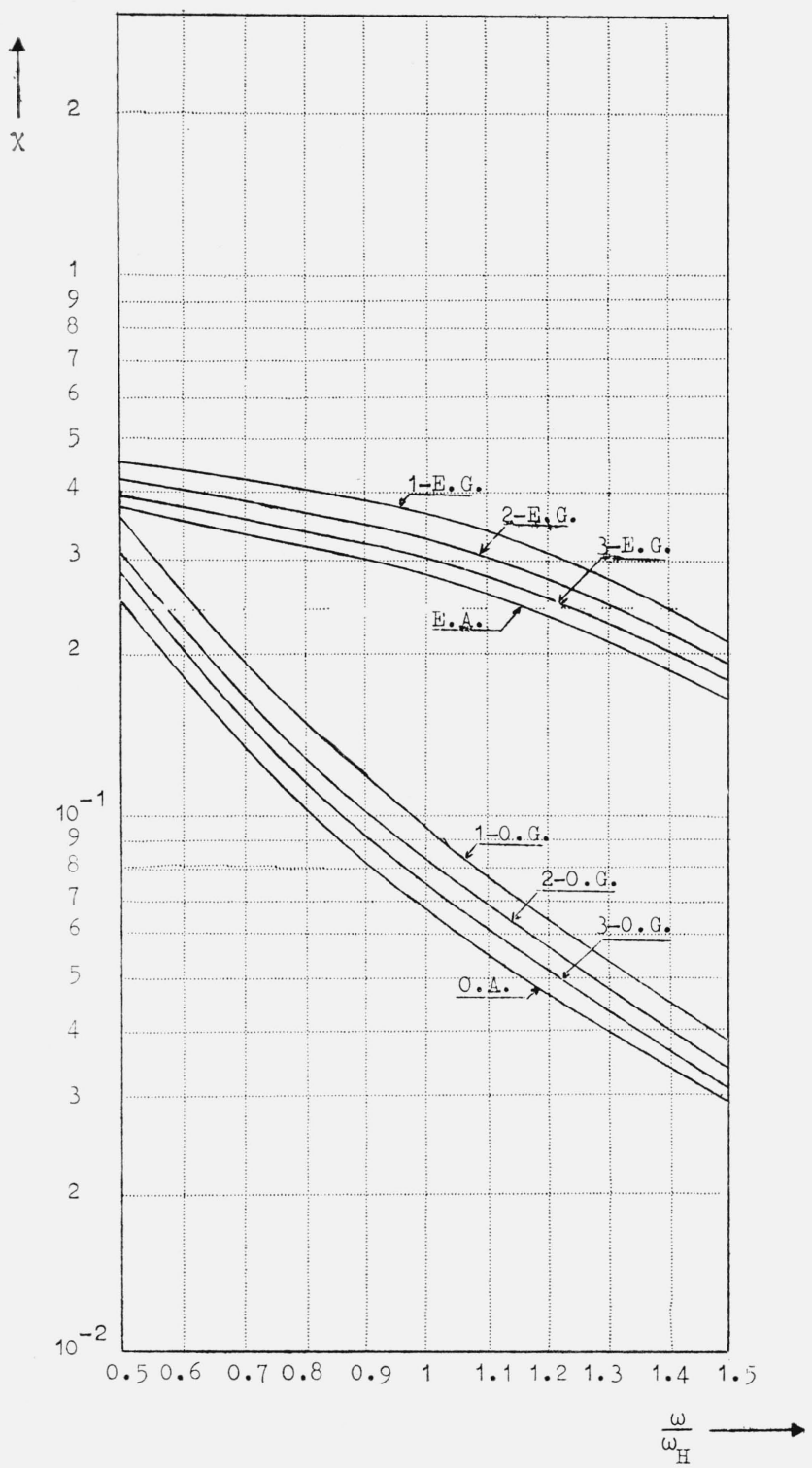

Figure 7. Values of $\chi$ for $\nu=7.5 \cdot 10^{6} \mathrm{coll} / \mathrm{sec}$. Symbols same as in figure 5 $a_{1 \max }=0.32 \quad a_{2 \max }=0.18 \quad a_{3 \max }=0.08$

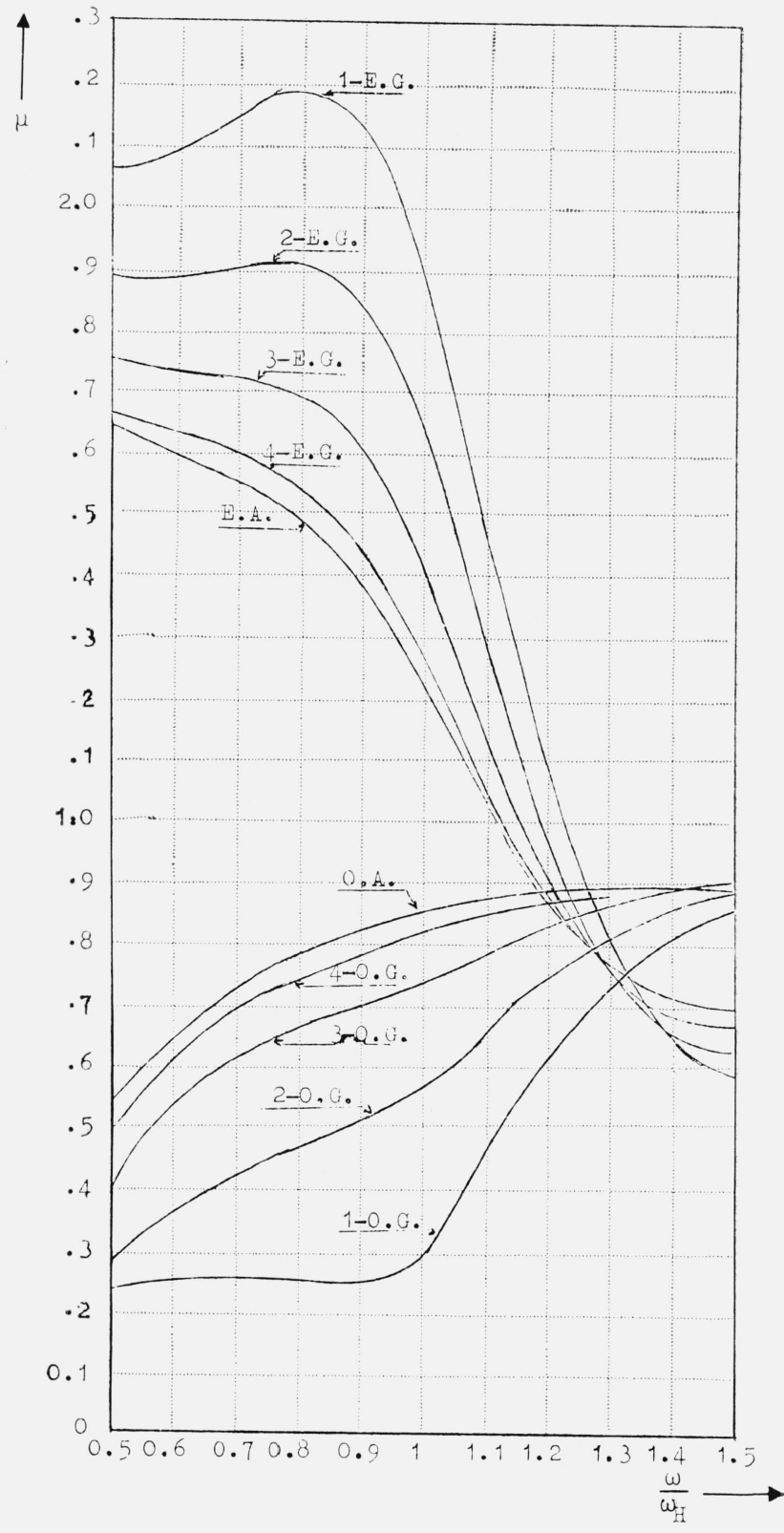

Figure 8. Values of $\mu$ for $\nu=2.5 \cdot 10^{6} \mathrm{coll} / \mathrm{sec}$. Symbols same as in figure 5 . $a_{1 \mathrm{max}}=2.49 \quad a_{2 \max }=1.40 \quad a_{3 \max }=0.62 \quad a_{4 \mathrm{max}}=0.15$

From figures 8 and 9 it can be seen that with increasing nonlinearity also the wave number of the O.G. wave increases. This fact generalizes a preceding result [Epstein, 1962] according to which with increasing nonlinearity, the wave number of an electromagnetic wave passing through an isotropic plasma is also increased.

The value of $a$, given by (28) varies along the curves traced in the figures, for every curve the maximum value of this quantity is indicated that, in general, is achieved by $a$ for $\omega \simeq \omega_{\mathrm{H}}$. It is seen that for some propagation cases the condition (28.1) is not fulfilled. For this reason it is interesting to see if we obtain different results takıng into account other terms in the development of $f_{00}$ in series of $E_{0}^{2}$. To this aim we have calculated the third term of the ex- 
pansion that gives for $A$ the value

$$
A=1+a+\frac{a^{2}}{6}
$$

and the fourth term for which

$$
A=1+a+\frac{a^{2}}{6}-\frac{a^{3}}{54}
$$
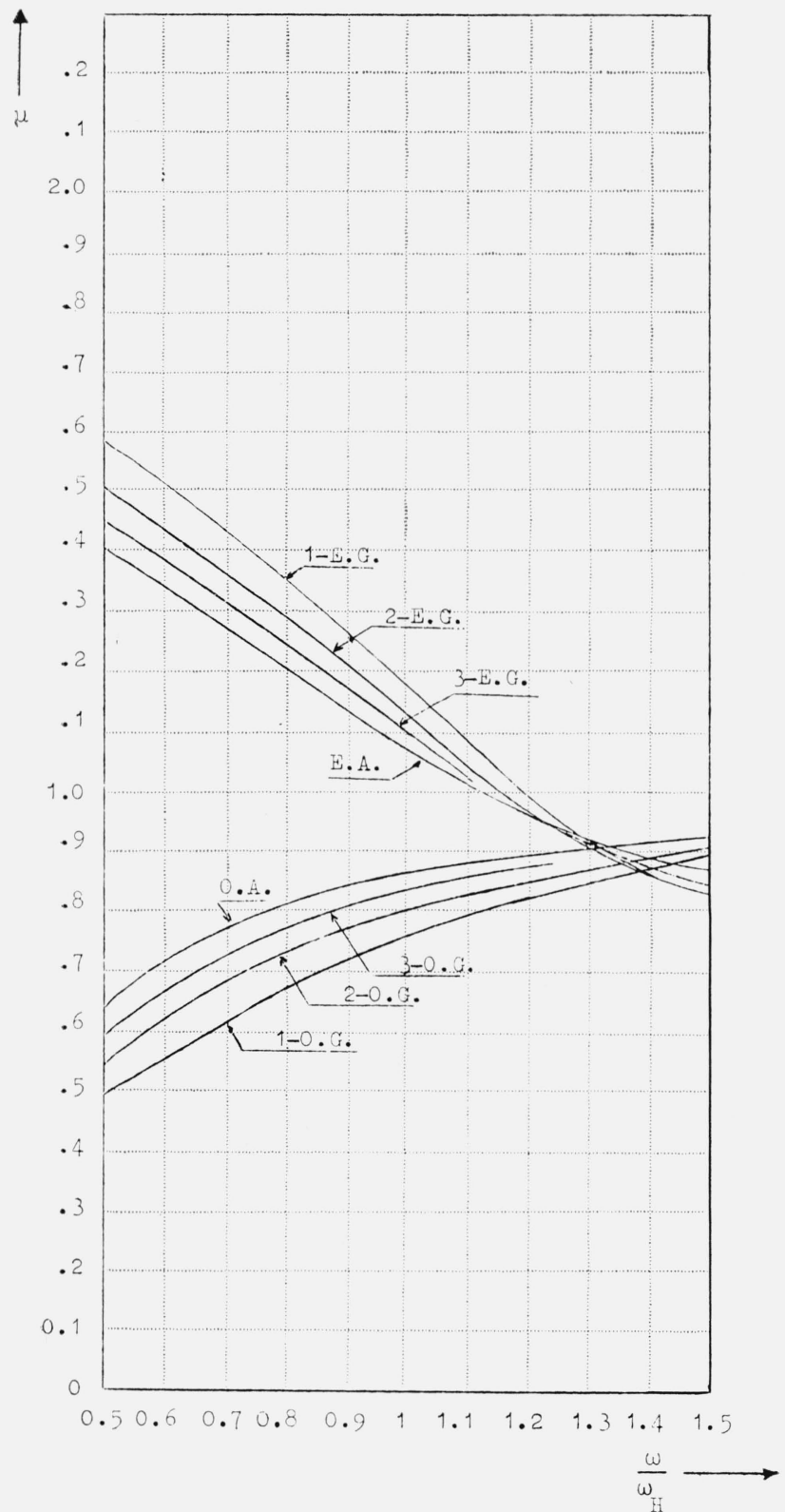

Figure 9. Values of $\mu$ for $\nu=5 \cdot 10^{6} \mathrm{coll} / \mathrm{sec}$. Symbols same as in figure 5 .

$a_{1 \mathrm{mgx}}=0.62 \quad a_{2 \mathrm{mgx}}=0.38 \quad a_{3 \mathrm{msx}}=0.17$

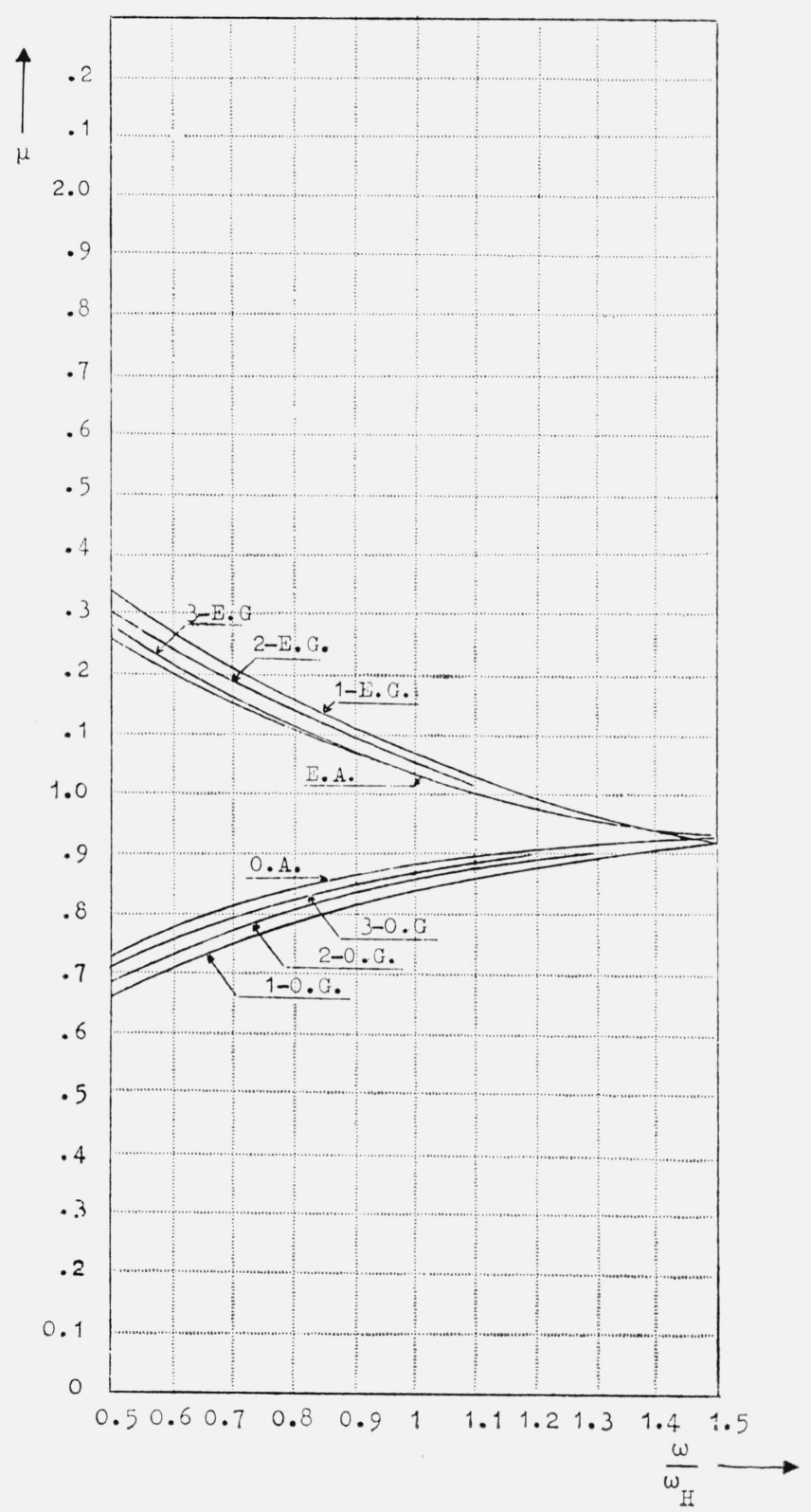

Figure 10. Values of $\mu$ for $\nu=7.5 \cdot 10^{6} \mathrm{coll} / \mathrm{sec}$. Symbols same as in figure 5 .

$a_{1 \mathrm{msx}}=0.32 \quad a_{2 \mathrm{mBx}}=0.18 \quad a_{3 \mathrm{msx}}=0.08$ 
If we consider the method used to calculate (31) we see immediately that we obtain the new dispersion relations taking for $A$ the values given by (33) and (34). The figures 11, 12, 13, and 14 show the results obtained in this way. For electric fields equal to, or less than, $10^{-4}$ volt $/ \mathrm{cm}$, the results calculated by means of the values of $A$ given by (29), (33), and (34) are practically equal. For stronger electric fields, of the order of $10^{-3}$ volt $/ \mathrm{cm}$, or for low collision frequencies (fig. 11 and 13) the results are quite different. With increasing $\nu$ (figs. 12 and 14) these differences disappear, given that the nonlinearity of the medium is decreased for the reasons explained before.

The problem that arises when we want to treat strong fields and low values of the collision frequency does not consist in choosing a particular form for $A$ but in showing that, in this case, we can still use the dispersion relation. In fact, as it can be seen from figure 8 it is possible to find some propagation conditions for which there is a strong difference between the phase velocities of the O.G. and E.G. waves. This causes, of course, a strong deformation of the monochromatic wave launched in the plasma. In this case we have harmonic generation and (25) and (30) loose their meaning. From this analysis we infer that it is not possible to treat, using a dispersion relation, the problem of nonlinear propagation of a rather strong electromagnetic wave in a plasma with a rather low value of the electronic collision frequency. We think that this problem could be solved in a satisfactory way by trying to integrate directly the system constituted by the equations of Boltzmann and of Maxwell. In fact, it is clear, that the electronic distribution function (15) from which we started in order to obtain (31) is valid only if the wave remains monochromatic.

\section{Electronic Distribution Function for an Amplitude Modulated Electric Field ${ }^{6}$}

The system of equations that we must integrate is given by (9) and (10), that is,

$$
\begin{gathered}
\frac{\partial f_{0}}{\partial t}+\frac{e}{3 m v^{2}} \frac{\partial}{\partial v}\left\{v^{2} \mathbf{E} \cdot \mathbf{f}_{1}\right\}-\frac{\delta}{2 v^{2}} \frac{\partial}{\partial v}\left[\nu v^{3}\left(1+\frac{k T}{m v} \frac{\partial}{\partial v}\right) f_{0}\right]=0 \\
\frac{\partial \mathbf{f}_{1}}{\partial t}+\frac{e}{m} \frac{\partial f_{0}}{\partial v} \mathbf{E}+\frac{e}{m c} \mathcal{H} \times \mathbf{f}_{1}+\nu \mathbf{f}_{1}=0
\end{gathered}
$$

where now

$$
\mathbf{E}=\mathbf{E}_{0}[1+\eta \cos (\alpha t+\beta)] \cos \omega t
$$

with $\omega_{H} \simeq \omega>>\alpha, 0 \leq \eta<1,0 \leq \beta<2 \pi$. We begin by defining three relaxation times: the first is the quick relaxation time of the electric field $\tau_{\text {E.q. }}$, it is determined by the frequency of the carrier and is of the order of $\omega^{-1}$, that is, $\tau_{\mathrm{E} . \mathrm{q} .} \sim \omega^{-1}$; the second is the slow relaxation time of the electric field $\tau_{\text {E.s. }}$, it is determined by the frequency of the modulating signal, and is of the order of $\alpha^{-1}$, that is, $\tau_{\mathrm{E} . \mathrm{s} .} \sim \alpha^{-1}$; the third is the relaxation time of the electronic energy $\tau_{r}$ that, as we know, is of the order of $(\delta \nu)^{-1}$. We shall suppose that

$$
\frac{1}{\omega_{H}} \simeq_{\tau_{\text {E. q. }}}<<\tau_{r}<<\tau_{\text {E. s. }} .
$$

If we remember the calculations of section 2 we see that (36) can be satisfied for values of $\alpha$ till to some kc/s. From the fact that $\tau_{\text {E.s. }}>>\tau_{r}$ derives that now the electronic distribution function must depend explicitly on time by means of the function $\cos (\alpha t+\beta)$. In this way we have the first term of $(9)$ of the order of $f_{0} / \frac{1}{\alpha} \sim \frac{1}{\tau_{\mathrm{E} . \mathrm{s}}} \cdot f_{0}$. Let us put, as for the monochromatic wave: ${ }^{7}$

$$
\mathbf{f}_{1}=-\mathbf{u} \frac{\partial f_{0}}{\partial v}
$$

\footnotetext{
${ }^{6}$ All the results contained in this section will appear, in a work by one of us (O. D. B.), in a more complete and elaborate form.

${ }^{7}$ It is easy to see that this method of integration is tantamount to developing the functions $f_{0}$ and $\mathrm{f}_{1}$ in a double series of powers of the parameters $\left(\frac{\tau_{\text {E.q. }}}{\tau_{r}}\right)<<1$ and $\left(\frac{\tau_{\text {E.q. }}}{\tau_{\text {E.q. }}}\right)<<1$. We shall calculate the first terms of these developments.
} 


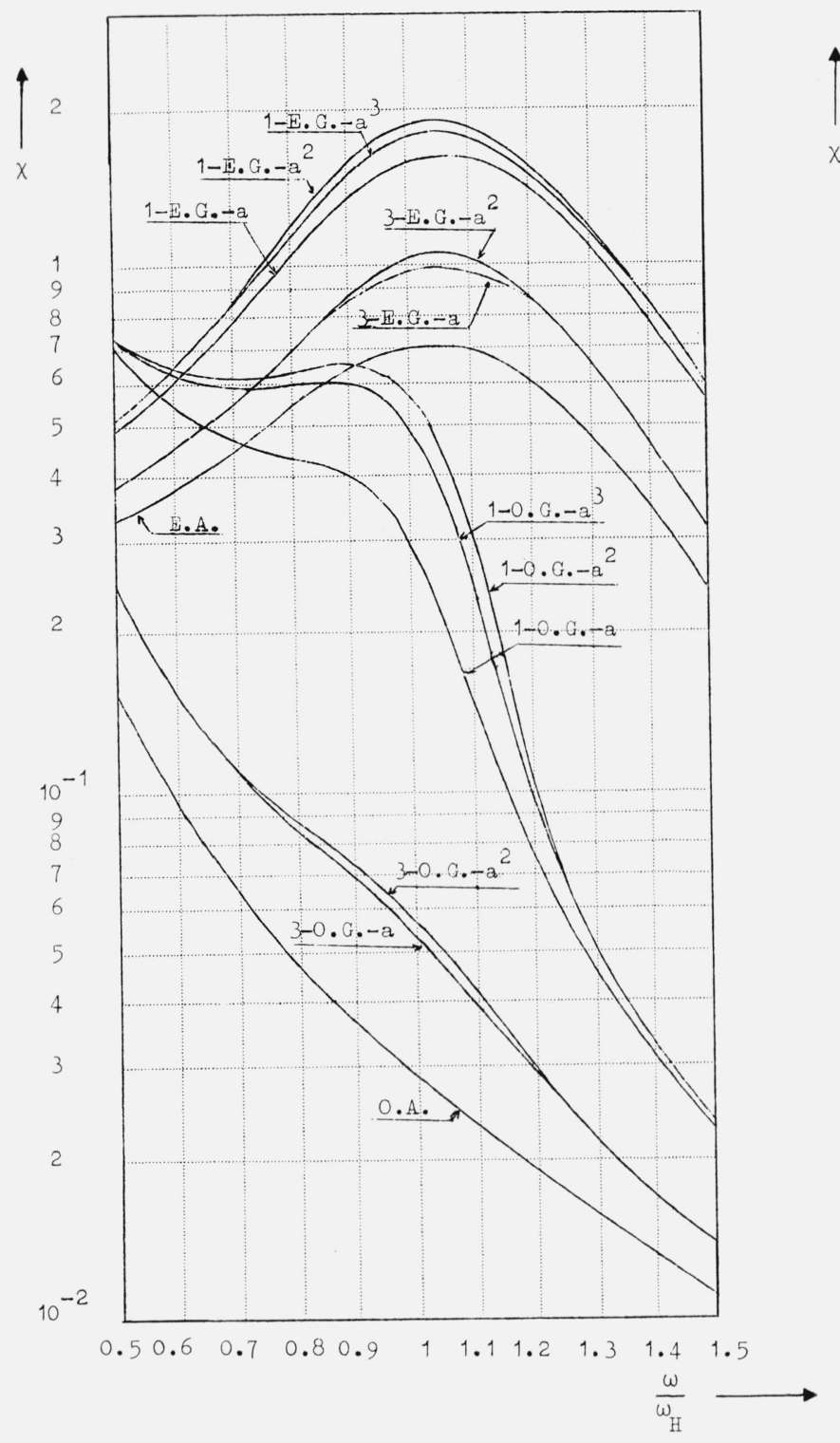

Figure 11. Values of $\chi$ for $\nu=2.5 \cdot 10^{6} \mathrm{coll} / \mathrm{sec}$. 1(O.G. and E.G.) $a$ with $A$ given by (29) $E_{0}=10^{-3} \mathrm{volt} / \mathrm{cm}$ 1(O.G. and E.G.) $a^{2}$ with $A$ given by (33) $E_{0}=10^{-3} \mathrm{volt} / \mathrm{cm}$ 1(O.G. and E.G.) $a^{3}$ with $A$ given by (34) $E_{0}=10^{-3}$ volt $/ \mathrm{cm}$ 3 (O.G. and E.G.) $a$ with $A$ given by (29) $E_{0}=5 \cdot 10^{-4}$ volt $/ \mathrm{cm}$ $3\left(\mathrm{O} . \mathrm{G}\right.$. and E.G.) $a^{2}$ with $A$ given by (33) $E_{0}=5 \cdot 10^{-4}$ volt $/ \mathrm{cm}$

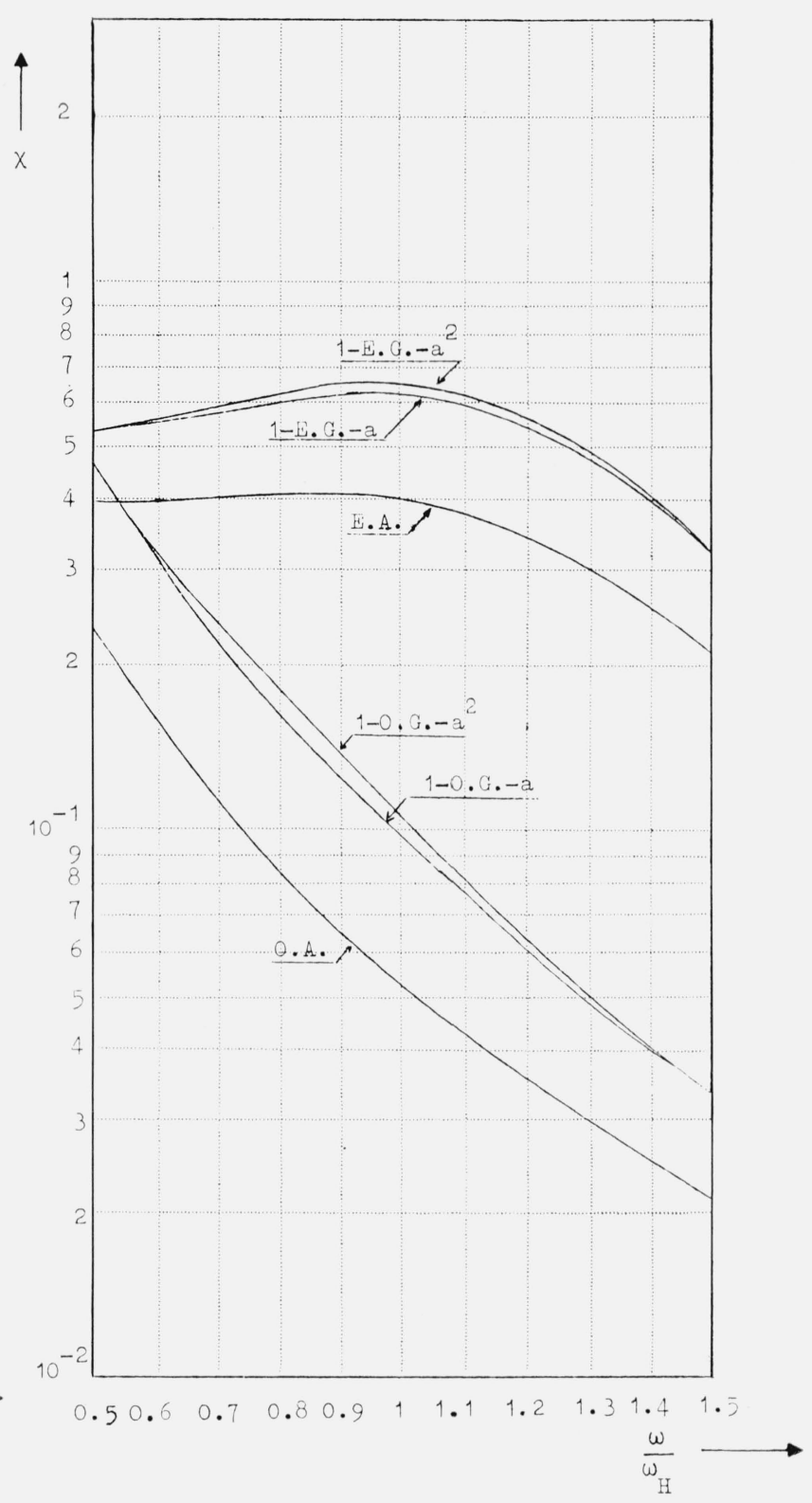

Figure 12. Values of $\chi$ for $\nu=5 \cdot 10^{6} \mathrm{coll} / \mathrm{sec}$. Symbols same as in figure 11.

We have

$$
\frac{\partial \mathbf{f}_{1}}{\partial t}=-\frac{\partial \mathbf{u}}{\partial t} \frac{\partial f_{0}}{\partial v}-\mathbf{u} \frac{\partial^{2} f_{0}}{\partial t \partial v}
$$

but $u$ (which gives the current) must vary in time at the rate of the frequency $\omega$ of the carrier, so $\partial \mathbf{u} / \partial t \simeq \omega \mathbf{u}$, in this way, disregarding terms of the order of $\alpha / \omega$, we have,

$$
\frac{\partial \mathbf{f}_{1}}{\partial t}=-\frac{\partial \mathbf{u}}{\partial t} \frac{\partial f_{0}}{\partial v}
$$

and (10) becomes the usual Langevin's equation,

$$
\frac{\partial u}{\partial t}+\nu \mathbf{u}=\frac{e}{m}\left[\mathbf{E}+\frac{\mathbf{u}}{c} \times \mathcal{H}\right]
$$




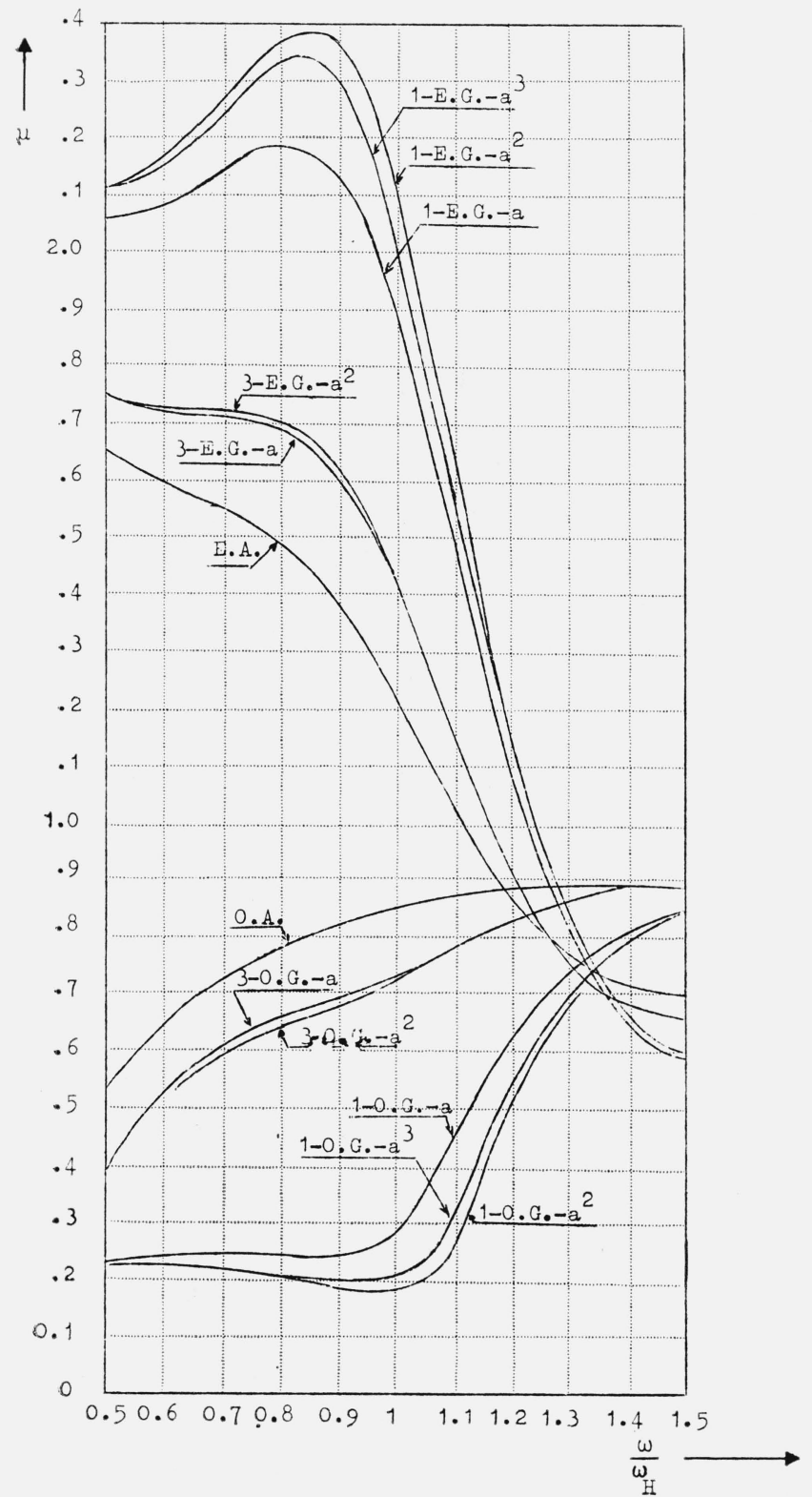

Figure 13. Values of $\mu$ for $\nu=2.5 \cdot 10^{6} \mathrm{coll} / \mathrm{sec}$. Symbols same as in figure 11 .

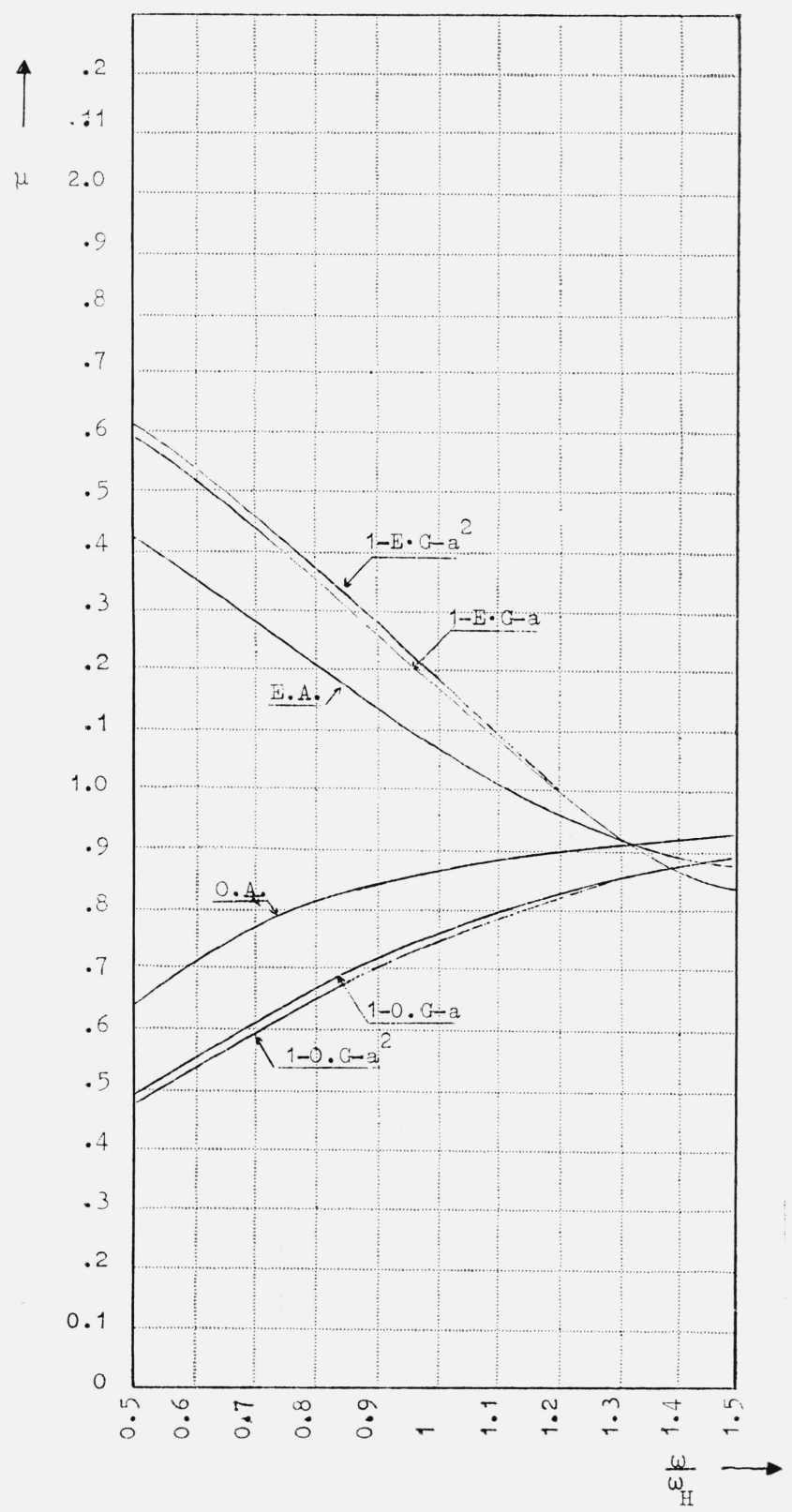

Figure 14. Values of $\mu$ for $\nu=5 \cdot 10^{6} \mathrm{coll} / \mathrm{sec}$. Symbols same as in figure 11.

We already know (see sec. (2)) the solution of this equation for a monochromatic wave, taking into account that (35) can be written as,

$$
\mathbf{E}=\mathbf{E}_{0} \frac{1}{2} \eta \cos \{(\omega+\alpha) t+\beta\}+\mathbf{E}_{0} \cos \omega t+\mathbf{E}_{0} \frac{1}{2} \eta \cos \{(\omega-\alpha) t-\beta\}
$$

and remembering (13) we have the persistent part of $\mathbf{f}_{1}$ given by:

$$
\left.\begin{array}{rl}
\mathbf{f}_{1}=[\mathbf{A}(\omega) & +\frac{1}{2} \eta\{\mathbf{A}(\omega+\alpha)+\mathbf{A}(\omega-\alpha)\} \cos (\alpha t+\beta) \\
& \left.+\frac{1}{2} \eta\{\mathbf{B}(\omega+\alpha)-\mathbf{B}(\omega-\alpha)\} \sin (\alpha t+\beta)\right] \frac{\partial f_{0}}{\partial v} \cos \omega t \\
+ & {\left[\mathbf{B}(\omega)+\frac{1}{2} \eta\{\mathbf{B}(\omega+\alpha)+\mathbf{B}(\omega-\alpha)\} \cos (\alpha t+\beta)\right.} \\
& \left.+\frac{1}{2} \eta\{\mathbf{A}(\omega-\alpha)-\mathbf{A}(\omega+\alpha)\} \sin (\alpha t+\beta)\right] \frac{\partial f_{0}}{\partial v} \sin \omega t
\end{array}\right\}
$$


In the calculation of $\mathbf{E} \cdot \mathbf{f}_{1}$ we disregard terms of the order of $\frac{\alpha}{\omega}=\frac{\tau_{\mathrm{E.q}} \text {, }}{\tau_{\mathrm{E} . \mathrm{s}}}$, that is, we put,

$$
\left.\begin{array}{l}
\mathbf{E}_{0} \cdot \mathbf{A}(\omega-\alpha)=\mathbf{E}_{0} \cdot \mathbf{A}(\omega)=\mathbf{E}_{0} \cdot \mathbf{A}(\omega+\alpha) \\
\mathbf{E}_{0} \cdot \mathbf{B}(\omega-\alpha)=\mathbf{E}_{0} \cdot \mathbf{B}(\omega)=\mathbf{E}_{0} \cdot \mathbf{B}(\omega+\alpha)
\end{array}\right\}
$$

and we define a function $\Xi(t)$ by:

in this way (9) becomes,

$$
\Xi(t)=[1+\eta \cos (\alpha t+\beta)]^{2}
$$

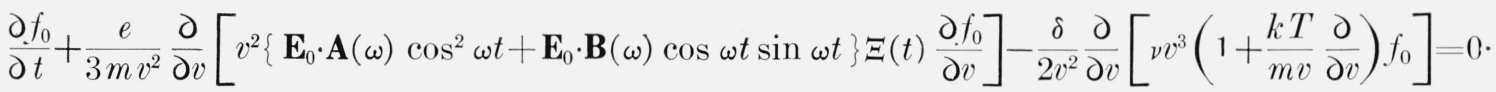

On account of (36) we see that the first term of this equation (of the order of $f_{0} / \tau_{\text {E.s. }}$ ) can be disregarded with respect to the second term (of the order of $f_{0} / \tau_{\text {E.q. }}$ ) and to the third term (of the order of $f_{0} / \tau_{r}$ ) so that taking the mean, over the period of the carrier, of the resulting equation we obtain,

$$
\frac{\delta}{2 v^{2}} \frac{\partial}{\partial v}\left[\nu v^{3}\left\{1+\left(\frac{k T}{m v}+\frac{e^{2} E_{0}^{2}}{3 m^{2} \delta v} \varphi(\nu) \Xi(t)\right) \frac{\partial}{\partial v}\right\} f_{0}\right]=0
$$

a first integration gives

$$
\nu v^{3} f_{0}+\nu v^{2}\left(k T+\frac{e^{2} E_{0}^{2}}{3 m \delta} \varphi(\nu) \Xi(t)\right) \frac{\partial f_{0}}{\partial v}=\Lambda(t)
$$

where $\Lambda(t)$ is an unknown function of time, it can be easily seen that, unless it is not identically zero, $f_{0}$ diverges strongly for $v=0$, so that $\Lambda(t) \equiv 0$ and

$$
f_{0}(v, t)=C(t) \exp \left\{-\int_{0}^{v} \frac{m v d v}{k T+\frac{e^{2} E_{0}^{2}}{3 m \delta} \varphi(\nu) \Xi(t)}\right\} .
$$

The study of this distribution function is made easy by the fact that it is identical with (15) provided that we substitute $E_{0}^{2}$ with $E_{0}^{2} \Xi(t)$. In particular we have the mean energy $\langle\epsilon\rangle$ depending on time, $\langle\epsilon(t)\rangle,{ }^{8}$ and taking as before only the wave $E_{\perp 0}^{-}$it is given by (19) where now

$$
\gamma=\frac{e^{2} E_{\perp 0}^{-2} \lambda^{2}}{3 \delta k^{2} T^{2}} \Xi(t)
$$

furthermore we have, as before,

$$
\left(\frac{\partial\langle\epsilon(t)\rangle}{\partial \omega}\right)_{\omega=\omega_{H}}=0
$$

Taking into account (38), (37) for $\mathbf{f}_{1}$ can be simplified in the following way

$$
\mathbf{f}_{1}=[1+\eta \cos (\alpha t+\beta)][\mathbf{A}(w) \cos \omega t+\mathbf{B}(w) \sin \omega t] \frac{\partial f_{0}}{\partial v}
$$

furthermore putting,

$$
\mathbf{j}_{t}=\frac{4 \pi}{3} e n \int_{0}^{\infty} v^{3} \mathbf{f}_{1} d v=\underset{=}{\sigma} \cdot \mathbf{E}+\frac{\partial \mathbf{P}}{\partial t}
$$

it is possible to evaluate the conductivity and the dielectric permittivity of the plasma. It is 
easy to see that disregarding terms of the order of $\frac{\alpha}{\omega}=\frac{\tau_{\text {E.q. }}}{\tau_{\text {E. s. }}}$ the components of these tensors are given by (24) provided that we substitute $E_{0}^{2}$ with $E_{0}^{2} \Xi(t)$. We can still use approximation (26), so that the components of the complex dielectric permittivity tensor can be written as

$$
\epsilon_{i k}^{\prime}(\omega, \alpha, t)=\epsilon_{i k}^{\prime}(\omega)+\Delta_{i k}(\omega, \alpha, t)
$$

being

$$
\Delta_{i k}(\omega, \alpha, t)=\epsilon_{i k}^{\prime}(\omega) \frac{e^{2} E_{0}^{2} \varphi(\nu)}{2 m \delta k T} \Xi(t)
$$

where $\epsilon_{i k}^{\prime}(\omega)$ is the expression that we obtain in the linear theory for the carrier. By (41) we see that $\epsilon_{i k}^{\prime}$ is varied, around its steady value $\epsilon_{i k}^{\prime}(\omega)$, by the quantity $\Delta_{i k}(\omega, \alpha, t)$. Let us now

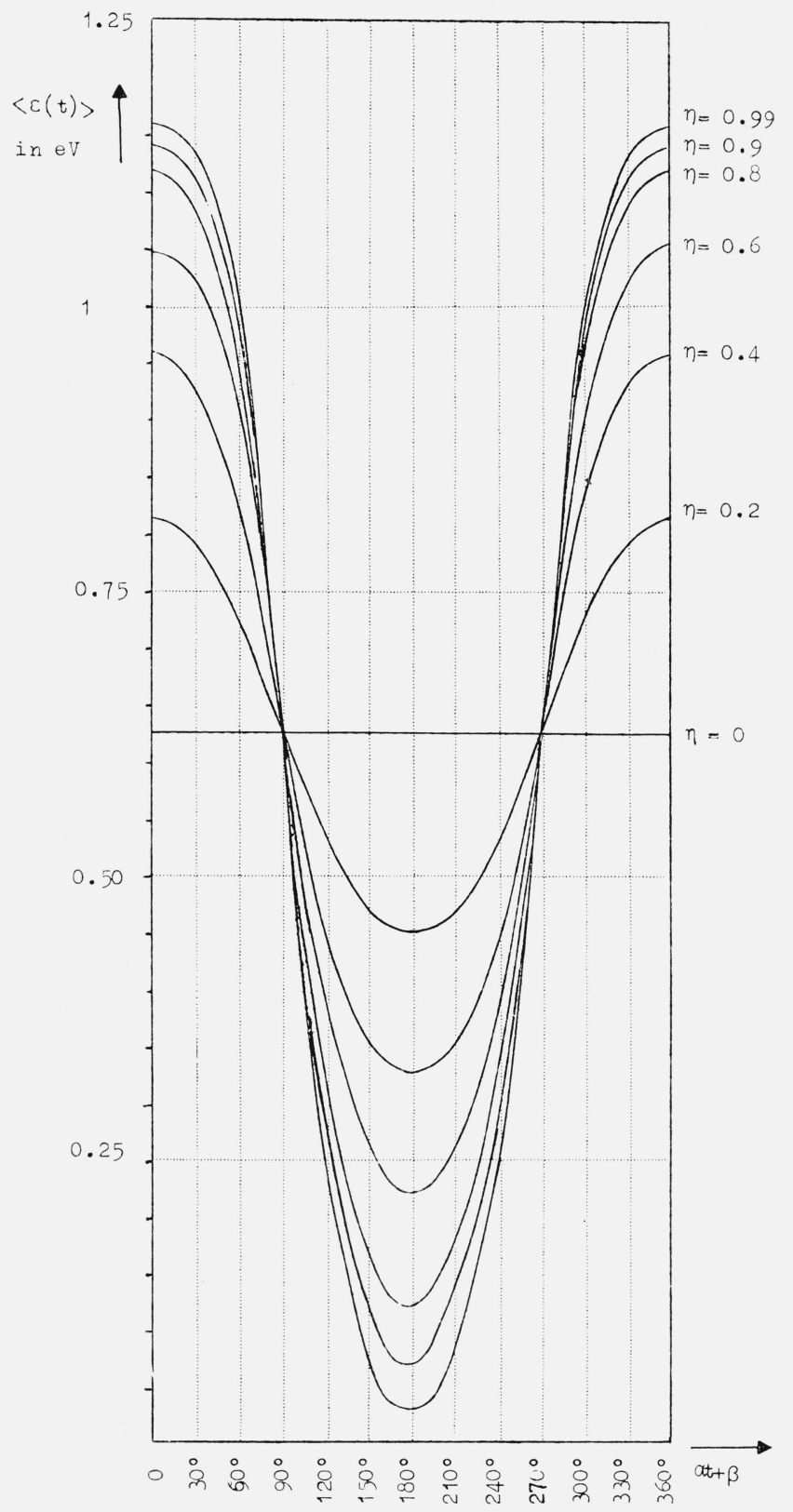

Figure 15. Behavior of $\langle\epsilon(\mathrm{t})\rangle$ for $\mathrm{E}_{\perp_{\mathrm{e}}}^{-}=$ $5 \cdot 10^{-4} \mathrm{volt} / \mathrm{cm}$.

Other constants same as in figure 1 , for various values of $\eta$. 
launch in the plasma, besides the wave (35), also another wave of the type,

$$
\mathbf{E}_{\gamma}=\mathbf{E}_{0 \gamma} \cos (\gamma t+\Lambda)
$$

Indicating with $\varphi(\omega, \nu)$ the function given by (14) and with $\varphi(\gamma, \nu)$ the same function with $\omega$ replaced by $\gamma$ and where $\Phi$ is substituted with the angle between $\mathbf{E}_{0 \gamma}$ and $\mathcal{H}$, it is easily seen that the electronic distribution function $f_{0}(v, t)$ is either:

$$
f_{0}(v, t)=C(t) \exp \left\{-\int_{0}^{v} \frac{m v d v}{k T+\frac{e^{2}}{3 m \delta}\left[E_{0}^{2} \varphi(\omega, \nu) \Xi(t)+2 E_{0 \gamma}^{2} \varphi(\gamma, v) \cos ^{2}(\gamma t+\Lambda)\right]}\right\}
$$

or

$$
f_{0}(v, t)=C(t) \exp \left\{-\int_{0}^{v} \frac{m v d v}{k T+\frac{e^{2}}{3 m \delta}\left[E_{0}^{2} \varphi(\omega, \nu) \Xi(t)+E_{0 \gamma}^{2} \varphi(\gamma, \nu)\right]}\right\} \text {. }
$$

Equation (43.1) is valid if $\tau_{E}>>\tau_{r}$, (43.2) is valid if $\tau_{E}<\left\langle\tau_{r}, \tau_{E}\right.$ being the relaxation time of the electric field (42) which is : $\tau_{E} \simeq \gamma^{-1}$. Furthermore it must be noticed that in the calculation of (43.1 and 43.2) we have neglected terms proportional to the product of the two waves (35) and (42); it is possible to make this approximation if there is no phase correlation between the two fields. Let us suppose now that the electric field (42) is small and that $\gamma$ is far from the gyromagnetic frequency so that we can neglect in (43) the part due to this wave, in this way $f_{0}(v, t)$ is given by $(40)$. The equation for the propagation of the wave $(42)$ is,

$$
\boldsymbol{\nabla} \times \boldsymbol{\nabla} \times \mathbf{E}_{\gamma}+\frac{1}{c^{2}} \underline{\epsilon}=\frac{\partial^{2} \mathbf{E}_{\gamma}}{\partial t^{2}}+\frac{4 \pi}{c^{2}} \frac{\partial \mathbf{j}_{\text {cond }}}{\partial t}=0
$$

and given that the wave (35) contributes to $\mathbf{j}_{\text {cond }}$, the propagation conditions of the wave (42) will depend on those of wave (35). We can still use the approximation (26); an easy calculation shows that in this case (44) becomes,

$$
\boldsymbol{\nabla} \times \boldsymbol{\nabla} \times \mathbf{E}_{\gamma}+\frac{1}{c^{2}} \underline{\boldsymbol{\epsilon}}(\gamma) \cdot \frac{\partial^{2} \mathbf{E}_{\gamma}}{\partial t^{2}}+\frac{4 \pi}{c^{2}} \stackrel{\sigma}{=}(\gamma) \cdot \frac{\partial \mathbf{E}_{\gamma}}{\partial t}+\mathcal{J}=0
$$

where $\underline{\epsilon}(\gamma)$ and $\underline{\sigma}(\gamma)$ are the expressions that we obtain in the linear theory for the wave $(42)$ and where $\mathscr{f}$ is given by:

$$
\mathcal{f}=\frac{4 \pi}{c^{2}}\left[1+\frac{e^{2} E_{0}^{2} \varphi(w, \nu)}{2 m \delta k T} \Xi(t)\right] \underset{=}{\sigma}(\omega) \cdot \frac{\partial \mathbf{E}}{\partial t} .
$$

It is clear that the wave (42) does not remain monochromatic and that these considerations constitute the starting point for a microscopic theory of cross modulation.

This paper presents some results of a research program in progress at the Istituto di Scienze Fisiche dell'Università di Milano with the contribution of C. N. R.

\section{References}

Budden, K. G. (1961), Radio Waves in the Ionosphere (Cambridge University Press, New York, N.Y.). Chapman, S., and T. G. Cowling (1960), The Mathematical Theory of Non-Uniform Gases (Cambridge University Press, New York, N.Y.).

Crompton, R. W., L. G. Huxley, and D. J. Sutton (1953), Experimental studies of the motions of slow electrons in air with application to the ionosphere, Proc. Roy. Soc. A218, 507. 
Davydov, B. J. (1936), Zurn. Eksp. Teor. Fiz. (U.S.S.R.) 6, 463.

Davydov, B. J. (1937), Zurn. Eksp. Teor. Fiz. (U.S.S.R.) $\boldsymbol{\gamma} 1069$.

Druyvesteyn, M. J., and F. M. Penning (1940), Rev. Mod. Phys. 12, 87.

Epstein, M. (1962), Electromagnetic-wave propagation in a plasma with nonlinear electrical conductivity. Phys. Fluids 5, 492.

Gallet, R. (1963), Whistlers, Geophysics, the Earth's Environment, ed. DeWitt, Hieblot, and Lebeau (Gordon and Breach, Publishers, Inc., New York, N.Y.).

Ginzburg, V. L., and A. V. Gurevich (1960), Nonlinear phenomena in a plasma located in an alternating electromagnetic field, Soviet Physics, Uspekhi 3, 115, 175.

Gurevich, A. V. (1957), Sov. Phys. JETP 3, 895.

Huxley, L. G. H. (1937), Phil. Mag. 23, 210, 442.

Huxley, L. G. H. (1940), Phil. Mag. 29, 313.

Huxley, L. G. H. (1951), Proc. Phys. Soc. 64B, 844.

Huxley, L. G. H., and R. W. Crompton (1962), The motion of slow electrons in gases, Atomic and Molecular Processes, ed. D. R. Bates (Academic Press, London, England and New York, N.Y.).

Kovrizhnikh, L. M. (1960), Effect of inelastic collisions on the velocity distribution of electrons, Sov. Phys. JETP 10, 347.

Phelps, A. V. (1960), J. Appl. Phys. 31, 1723.

Sen, H. K., and A. A. Wyller (1960), On the generalization of the Appleton-Hartree magneto-ionic formulas, J. Geophys. Res. 65, No. 12, 3931.

Smit, J. A. (1936), Physica 3, 543.

Whittaker, E. T., and G. N. Watson (1958), Modern Analysis (Cambridge University Press, New York ,N.Y.).

(Paper 69D1-441) 